\title{
Visuospatial Coding in Primate Prefrontal Neurons Revealed by Oculomotor Paradigms
}

\author{
SHINTARO FUNAHASHI, CHARLES J. BRUCE, AND PATRICIA S. GOLDMAN-RAKIC \\ Section of Neuroanatomy, Yale University School of Medicine, New Haven, Connecticut 06510
}

\section{SUMMARY AND CONCLUSIONS}

1. Visual responses and their relationship to delay-period activity were studied by recording single neuron activity from the prefrontal cortex of rhesus monkeys while they performed an oculomotor delayed-response (ODR) and a visual probe (VP) task. In the ODR task, the monkey was required to maintain fixation of a central spot of light throughout the cue $(0.5 \mathrm{~s})$ and delay ( $3 \mathrm{~s}$ ) periods and then make a saccadic eye movement to one of four or eight locations where the visual cue had been presented. In the VP task, the same visual stimuli that were used in the ODR task were presented for $0.5 \mathrm{~s}$, but no response was required. The VP task was thus employed to test the passive visual response and, by comparison with cue-elicited activity in the ODR task, to examine the degree of behavioral enhancement present in prefrontal visual activity.

2. Among 434 neurons recorded from the prefrontal cortex within and surrounding the principal sulcus (PS), 261 had taskrelated activity during at least one phase of the ODR task, and 74 of these had phasic visual responses to the onset of the visual cues with a median latency of $116 \mathrm{~ms}$. The visual responses of 69 neurons were excitatory, and 5 neurons were inhibited. Five of the neurons with excitatory visual responses also responded transiently after the offset of the cue.

3. Visual responses were classified as directional for 71 PS neurons $(96 \%)$ in that excitatory or inhibitory responses occurred only for location of cues in a restricted portion of the visual field. Only 3 PS neurons were omnidirectional, i.e., responded equivalently to cues in all locations tested.

4. The best direction and tuning specificity of all PS neurons with directional visual responses were estimated from parameters yielding the best fit to a Gaussian-shaped tuning function. The best direction for the majority $(71 \%)$ of neurons was toward the visual field contralateral to the hemisphere where the neuron was located. The remaining neurons had their best directions in the ipsilateral field $(18 \%)$ or along the vertical meridian $(11 \%)$.

5. The specificity of directional tuning for PS visual responses was quite variable, ranging from neurons that responded only to one of the eight cue locations to neurons that responded to all eight, but in a clearly graded fashion. The standard deviation parameter of the Gaussian curve indexed the breadth of directional tuning of each neuron; its median value was $37^{\circ}$

6. Visual responses of 36 PS neurons were obtained in both the ODR task and the VP task. Extremely strong positive correlations were found for both best direction and tuning across the two tasks. suggesting that both tasks elicit the same underlying visual responses via receptive field activation.

7. To examine how visual responses in the prefrontal cortex are modified by their behavioral significance, enhancement indexes were calculated for the 36 PS neurons based on their responses to visual cues in the ODR task relative to their responses to the same stimuli in the VP task. The majority (79\%) of PS neurons showed enhancement indexes between 0.5 and 2.0, indi- cating an insignificant effect, and the median overall enhancement index was 1.12 .

8. Distinct populations of PS neurons carry visual and mnemonic information, including neurons with only cue-period activity ( $n=27)$, neurons with both cue- and delay-period activity $(n=33)$, and a large population of neurons $(n=78)$ having only delay-period activity. Composite histograms constructed for each of these three groups indicate that population delay-period activity across PS neurons builds gradually after a phasic response to the cue.

9. A comparison of the directional selectivity of the visual responses with that of the delay-period activation was made for 27 PS neurons with both types of activity in the ODR task. There was a high positive correlation in the best directions across neurons.

10. These findings indicate that visual inputs to the principal sulcal area play an important role in constructing "memory fields" that hold visuospatial information "on-line" when a stimulus goes out of view. However, the results also indicate that the mnemonic activity is not simply an enhancement in magnitude and duration of a neuron's visual responses. The mnemonic process may rather involve several distinct types of PS neurons that communicate with each other locally.

\section{INTRODUCTION}

Neuronal activity in the dorsolateral prefrontal cortex of the monkey has been extensively studied in conjunction with a variety of behavioral tasks and conditions. Neurons there have been shown to respond during manual delayedresponse tasks (Batuev et al. 1985; Fuster 1973; Kojima and Goldman-Rakic 1982, 1984; Kubota et al. 1974; Niki et al. 1974c, 1976), spatial alternation tasks (Batuev et al. 1981; Kubota and Niki 1971; Niki 1974a,b), conditional response tasks (Watanabe 1981), go/no-go tasks (Komatsu 1982; Watanabe 1986), visual discrimination tasks (Kubota et al. 1980; Fuster et al. 1982; Rosenkilde et al. 1981; Quintana et al. 1988; Yajeya et al. 1988), visual detection tasks (Mikami et al. 1982b), and several types of saccade tasks (Boch and Goldberg 1989; Joseph and Barone 1987; Kojima 1980) including oculomotor delayed-response (ODR) tasks (Funahashi et al. 1989a). Activity of prefrontal neurons under many of these complex behavioral conditions has been argued to reflect the role of the prefrontal cortex in spatial memory and other aspects of learned and cognitive behavior and to provide a basis for interpreting the effects of lesions of the prefrontal cortex on performance of these tasks in both humans and monkeys (Bruce 1988; Fuster 1989; Goldman-Rakic 1987; Stuss and Benson 1986).

One major category of neuronal activity in the prefrontal cortex is responsivity to sensory stimuli. This can take the 


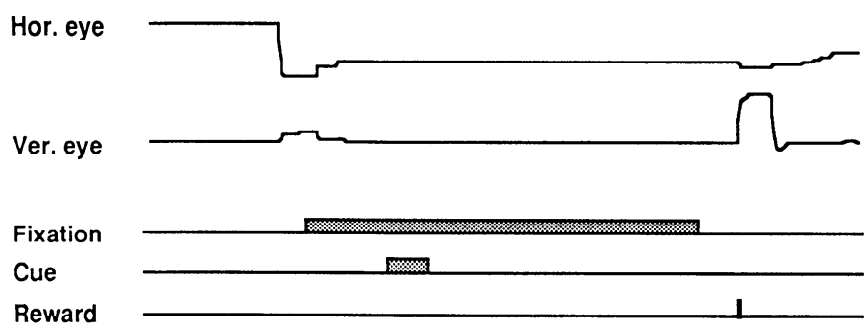

B Visual probe (VP) task

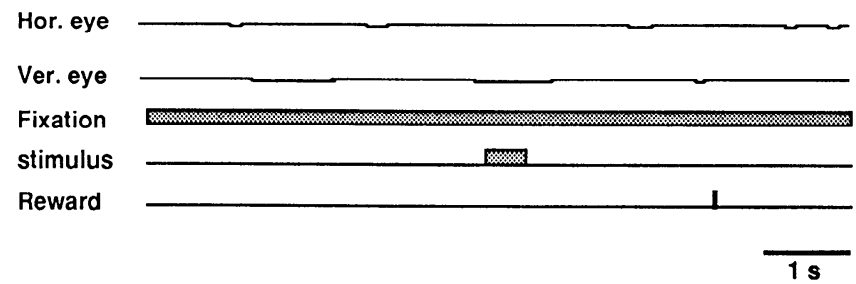

form of a response to the visual cue in a delayed-response task (Fuster 1973; Kojima 1980; Niki et al. 1974c, 1976). However, visual responses of prefrontal neurons can also be observed in tasks in which peripheral visual stimuli have no behavioral significance to the monkey (Ito 1982; Mikami et al. 1982a; Suzuki and Azuma 1983) and even during recordings from paralyzed (Pigarev et al. 1979) or anesthetized (Schechter and Murphy 1975) monkeys. Receptive fields of prefrontal neurons under these conditions are usually very large and primarily represent the visual field contralateral to the neuron being studied. Although some prefrontal neurons have preferences for complex visual stimuli (Pigarev et al. 1979), many, especially in the caudal half of the prefrontal cortex, respond well to a simple spot of light (Mikami et al. 1982a; Suzuki and Azuma 1983).

Because the behavioral tasks that have been examined are complex and usually involve multiple presentations of visual stimuli, the property of the stimulus to which a prefrontal neuron is responding during such tasks is often unclear. Because in many previous studies eye movements were not controlled, and the retinal locations of visual stimuli were not recorded, it is not clear how precisely prefrontal activity registers information about spatial location. Conversely, many studies test only a few spatial locations, and it is not known how the whole visual field is processed. In the present study, we have employed an ODR task in which the monkeys were trained to maintain fixation during presentation of stimuli at different locations across the visual field and during the subsequent delay period. With this method of controlling the retinotopic location of the visual cue, we could more accurately determine the spatial specificity of prefrontal activity.

In addition, we investigated whether the sensory response of prefrontal neurons could be modulated in accordance with its behavioral significance. An "enhancement" of sensory responses in conjunction with saccadic eye movement tasks has been demonstrated in the superior colliculus (Goldberg and Wurtz 1972; Wurtz and Mohler 1976a), the posterior parietal cortex (Bushnell et al. 1981;<smiles></smiles>

FIG. 1. Schematic drawings of behavioral paradigms. $A$ : oculomotor delayed-response task. $B$ : visual probe task. Horizontal and vertical eye positions and timing of visual stimulus presentations (fixation point, visual cue, and visual probe stimulus) and reward delivery are shown for each task.

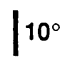

Robinson et al. 1978), the frontal eye fields (Bruce and Goldberg 1985; Goldberg and Bushnell 1981; Wurtz and Mohler 1976b), and the substantia nigra (Hikosaka and Wurtz 1983). The term enhancement refers to the phenomenon in which the identical physical stimulus elicits a significantly larger response from the recorded neuron when that stimulus has behavioral relevance as compared to the response it elicits when it is task-irrelevant. Only a few studies have explored enhancement in prefrontal neurons. Mikami et al. (1982b) found only minimal enhancement of visual responses in conjunction with a visual detection task versus a visual probe (VP) task. In the present study we compared responses of prefrontal neurons to visual stimuli during a VP task to those of the same neurons while exactly the same visual stimuli were presented in the context of the ODR task. One might expect an enhancement of visual activity in the context of this task inasmuch as the prefrontal cortex is essential for optimal performance of short-term spatial memory tasks, including the ODR task (Funahashi et al. 1989a).

Finally, we compared the visual activation of prefrontal neurons with their activity during the delay period of the ODR task. We have previously reported that many pre-

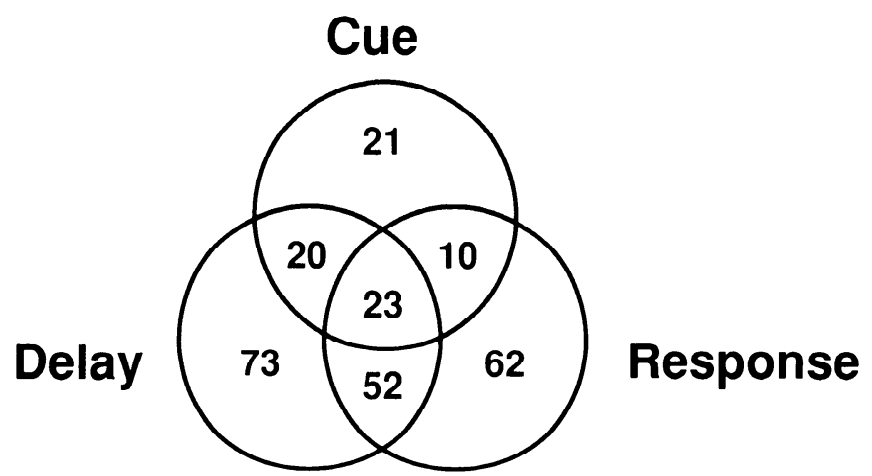

FIG. 2. Venn diagram showing incidence of principal sulcus neurons with cue-period activity, delay-period activity, response period activity, and combinations of these activities. 


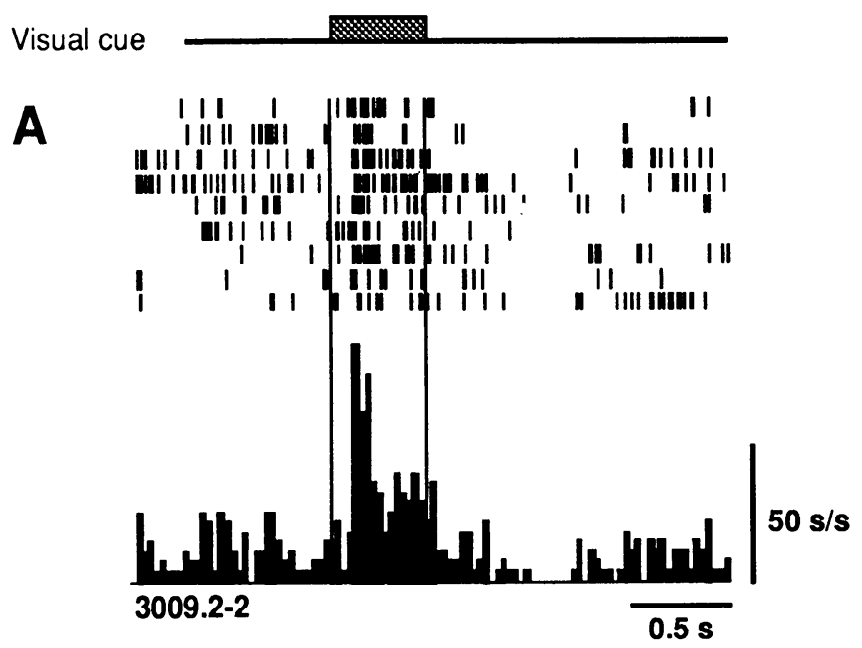

B
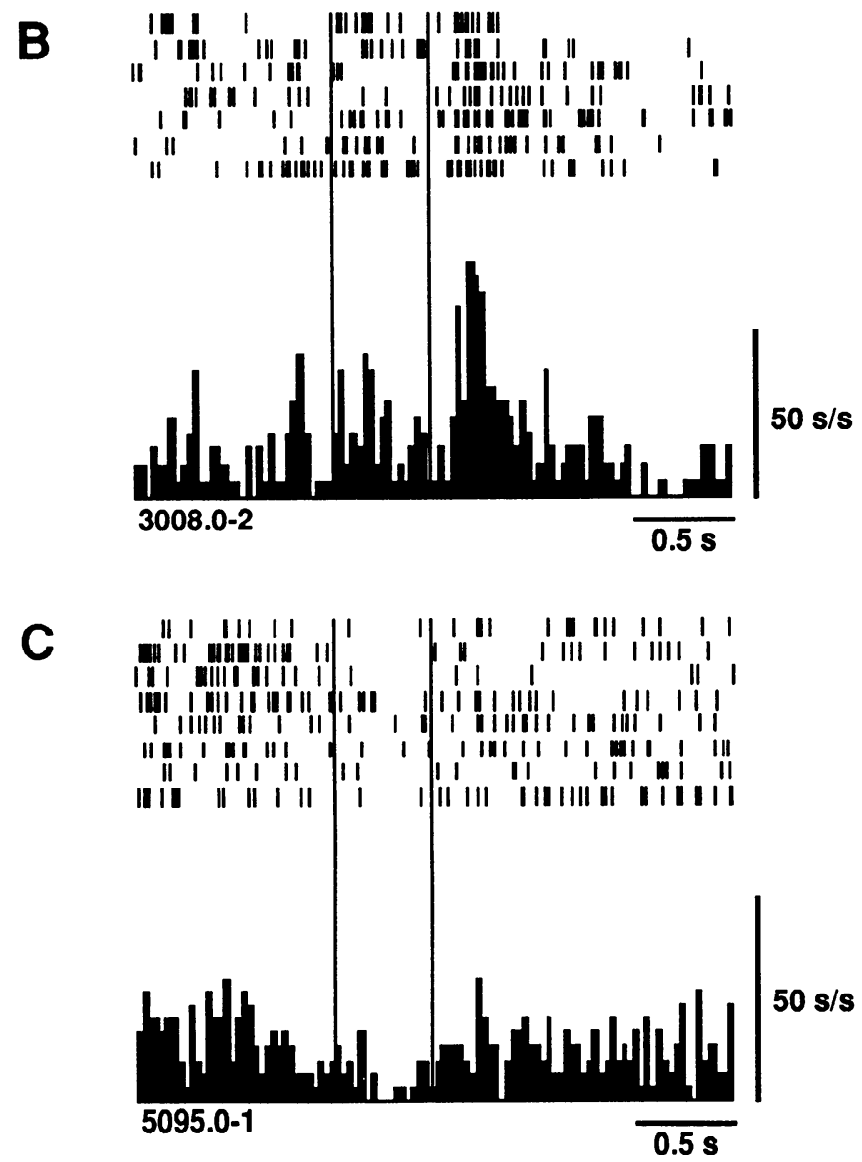

FIG. 3. Three types of responses to the visual cue in the oculomotor delayed-response task. $A$ : excitatory on-response. $B$ : excitatory off-response. $C$ : inhibitory on-response. Histogram binwidth is $30 \mathrm{~ms}$.

frontal neurons have "memory fields," i.e., show excitation or inhibition in the delay period only for selected, usually contralateral directions (Funahashi et al. 1989a). Knowledge about the degree of similarity between the memory field of a prefrontal neuron and its visual receptive field could provide insight into the issue of how memory fields are constructed in prefrontal neurons.

Preliminary results from this study have been presented in abstract form (Funahashi et al. 1989b).

\section{METHODS}

\section{Subjects and experimental setups}

Four rhesus monkeys (Macaca mulatta, $3.2-5.3 \mathrm{~kg}$ ) served as subjects. The experimental apparatus, surgical procedures, recording procedures, and basic methods of data analysis are described in detail in our previous report (Funahashi et al. 1989a). Briefly, the monkey sat in the primate chair during the experiment with his head fixed in position in a dark room. The monkey faced a 19-in cathode ray tube (CRT) (RCA, TC1119) subtending $48 \times 38^{\circ}$ in visual angle, where visual cues were presented using a GRAPH-1 1 graphics card (Pacific Binary Systems). There was no background illumination on the CRT. The fixation target was a small white spot $\left(0.1^{\circ}\right.$ diam $)$ usually presented at the center of the CRT. The peripheral visual cues were filled white squares $(0.7 \times$ $0.7^{\circ}$ ). The magnetic search coil technique (Robinson 1963) was used to monitor the monkey's eye position and movements. An on-line computer system (PDP-11) presented visual stimuli, monitored eye position, and rewarded correct oculomotor behavior with $0.2-\mathrm{ml}$ drops of lightly sweetened water. Water was not available at the monkey's home cage during recording period; instead they worked to satiety during the daily 2 - to $4-\mathrm{h}$ recording sessions and were given ad lib chow and fruit on return to their home cage.

\section{ODR task}

Figure $1 A$ shows a schematic drawing of the ODR task. After a 5-s intertrial interval (ITI), the fixation target appeared at the center of the CRT. The monkey looked at the fixation target and maintained fixation for $0.75 \mathrm{~s}$ (the fixation period), whereupon the visual cue was presented for $0.5 \mathrm{~s}$ (the cue period) at one of four or eight peripheral locations ( $13^{\circ}$ eccentricity). Stimulus location was randomized over trials so that the monkey could not predict where the cue would appear on any given trial. A crucial feature of the task was that the monkey had to maintain fixation throughout the cue period and also throughout the subsequent delay period (usually $3 \mathrm{~s}$ ). At the end of the delay, the fixation target was extinguished; this was the "go" signal to make a saccade. If the monkey made a saccadic eye movement within the next $0.5 \mathrm{~s}$ (the response period) to the location where the cue had been presented, it was rewarded with a drop of water. A correct response was defined as an eye movement that fell within a predetermined window $\left(6^{\circ}\right.$ in diameter) around the cue location.

\section{VP task}

In the VP task (Fig. $1 B$ ), the fixation target was present at the center of the CRT at all times, including the ITI, to prevent

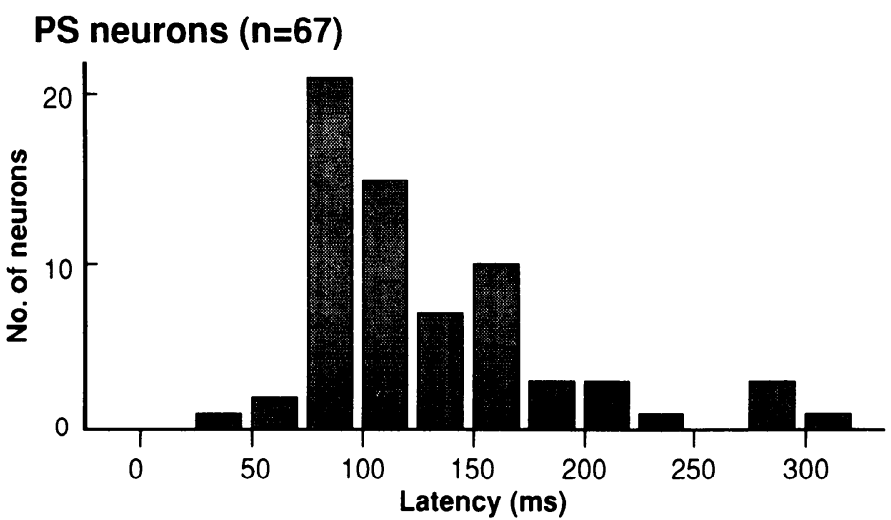

FIG. 4. Distribution of visual response latencies for principal sulcus neurons. The latency for each neuron was measured by making cumulative histograms at the cue direction yielding the maximum visual response. 


\section{A ODR task}
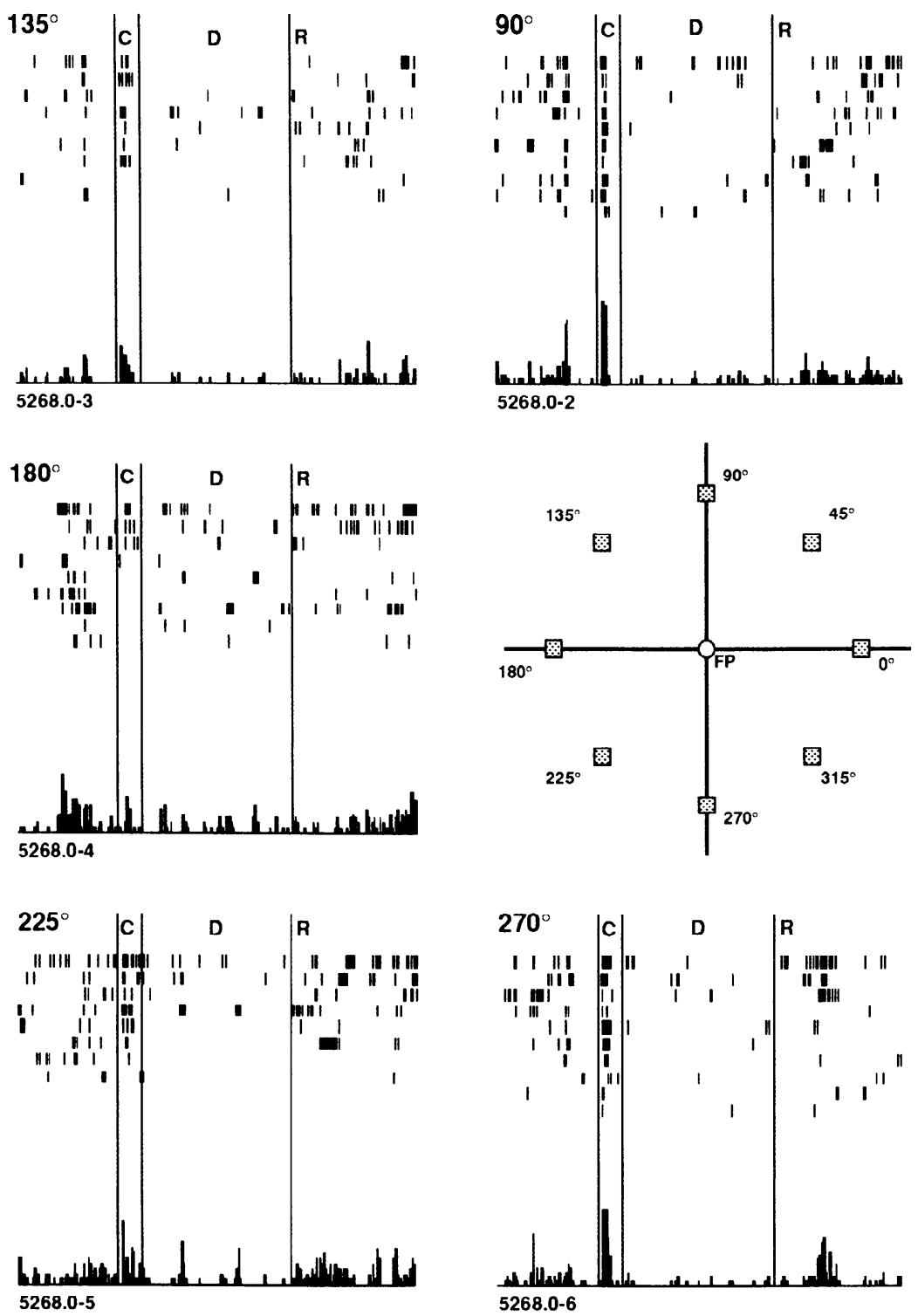
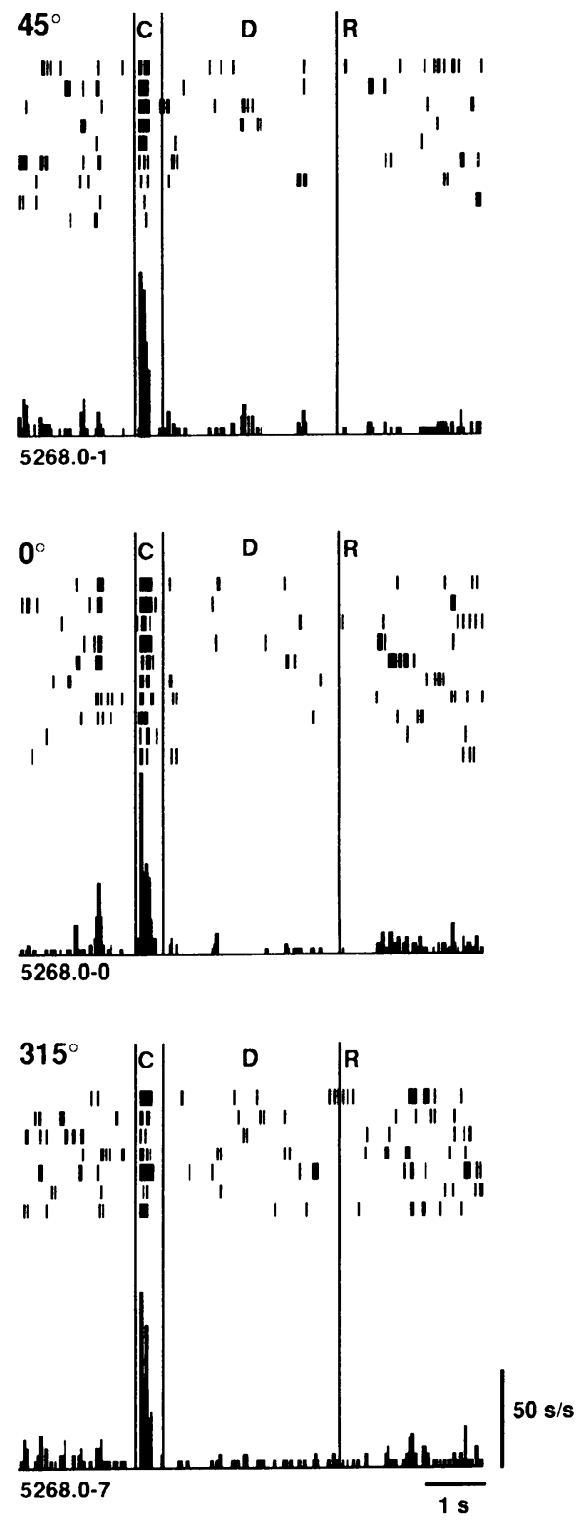

\section{B cue period activity}

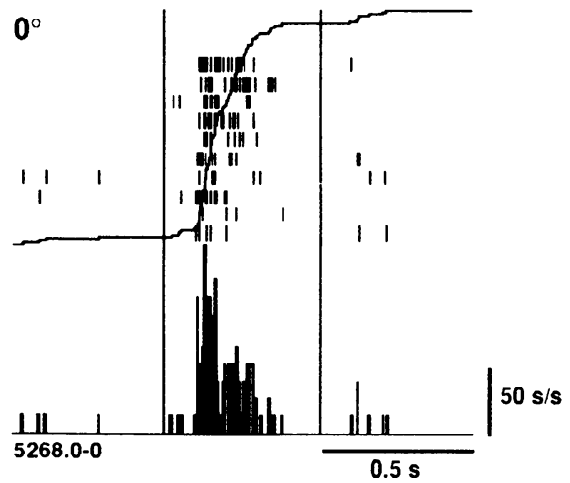

FIG. 5. A: dircctional cue-period activity of a principal sulcus neuron during the oculomotor delayed-response task. This neuron (5268.0, left hemisphere) had a strong phasic excitatory response to the onset of cues in the right visual field. Visual cues were randomly presented in 1 of the 8 locations indicated in the central diagram. All cue eccentricities were $13^{\circ}$, and all delay periods were $3 \mathrm{~s}$. Histogram binwidth is $40 \mathrm{~ms}$. B: cue-period activity at the $0^{\circ}$ trials of the same neuron $(5268.0)$ is shown with the time scale expanded and a cumulative histogram, in addition to the rasters and conventional histogram. Visual response latency at the $0^{\circ}$ location was $94 \mathrm{~ms}$ as judged by the inflection of the cumulative histogram. C, D, R, cue, delay, and response periods. Histogram binwidth is $15 \mathrm{~ms}$. 
neuron 5277

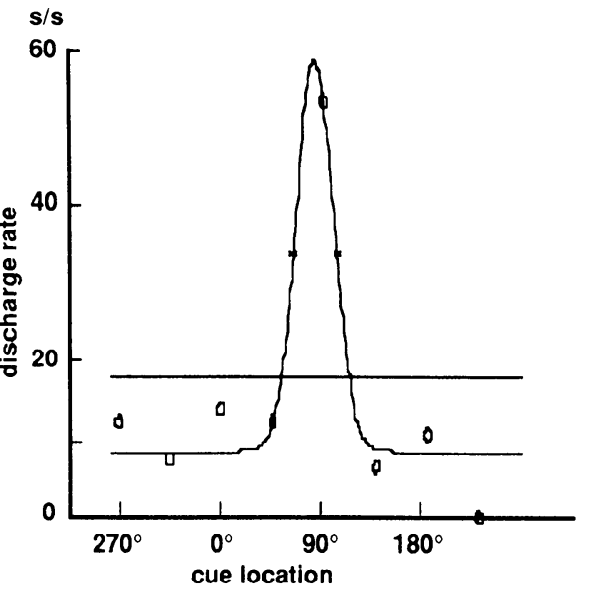

neuron 7074

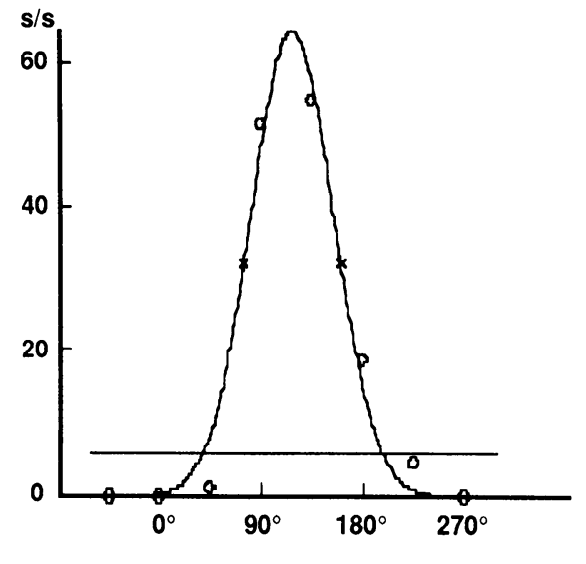

neuron 5268

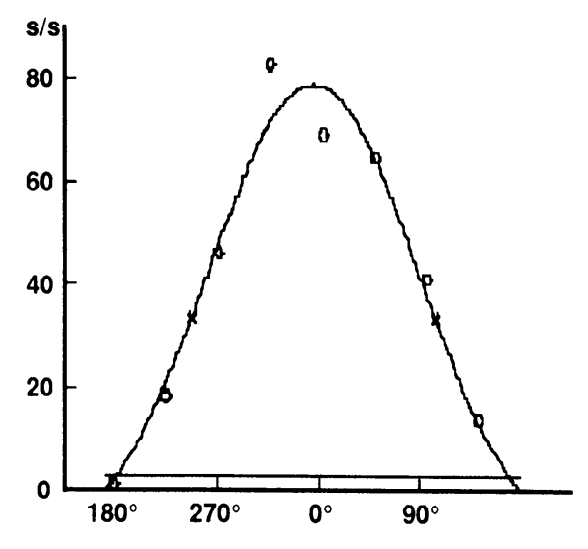

FIG. 6. Directional tuning of cue-period activity for 3 principal sulcus neurons in the oculomotor delayed response task. Plots show discharge rate during the cue period for the 8 different cue directions, with a Gaussian function fit to the data. Rate for each cue direction was measured during the 100-ms period, starting at the neuron's response latency in its best direction. Visual cues were presented in 1 of the 8 directions randomly, always at $13^{\circ}$ eccentricity, and each data point represents the average of 6-10 trials. Neuron 5277, left hemisphere, has narrowly tuned $\left(T_{d}=16^{\circ}\right)$ excitatory cue-period activity with its best direction at $82^{\circ}$. Neuron 7074 , right hemisphere, has moderately tuned $\left(T_{d}=37^{\circ}\right)$ excitatory cue-period activity with its best direction at $119^{\circ}$. Neuron 5268 , left hemisphere, has broadly tuned $\left(T_{d}=90^{\circ}\right)$ excitatory cue-period activity with its best direction at $352^{\circ}$. Horizontal lines in tuning curves indicate the neuron's spontaneous discharge rate.

saccadic eye movements to the visual target; the monkey was simply required to maintain fixation continuously to obtain reward. While the monkey maintained fixation, a peripheral visual test stimulus appeared for $0.5 \mathrm{~s}$ on the CRT. If the monkey maintained fixation, a reward was delivered $2 \mathrm{~s}$ after the offset of the test stimulus. If the monkey broke fixation during the presentation of test stimuli or during the subsequent 2-s fixation period, the test stimulus disappeared immediately and the task entered the ITI ( $3 \mathrm{~s})$ without reward. The visual stimuli and the location

\section{PS neurons $(\mathrm{n}=62)$}

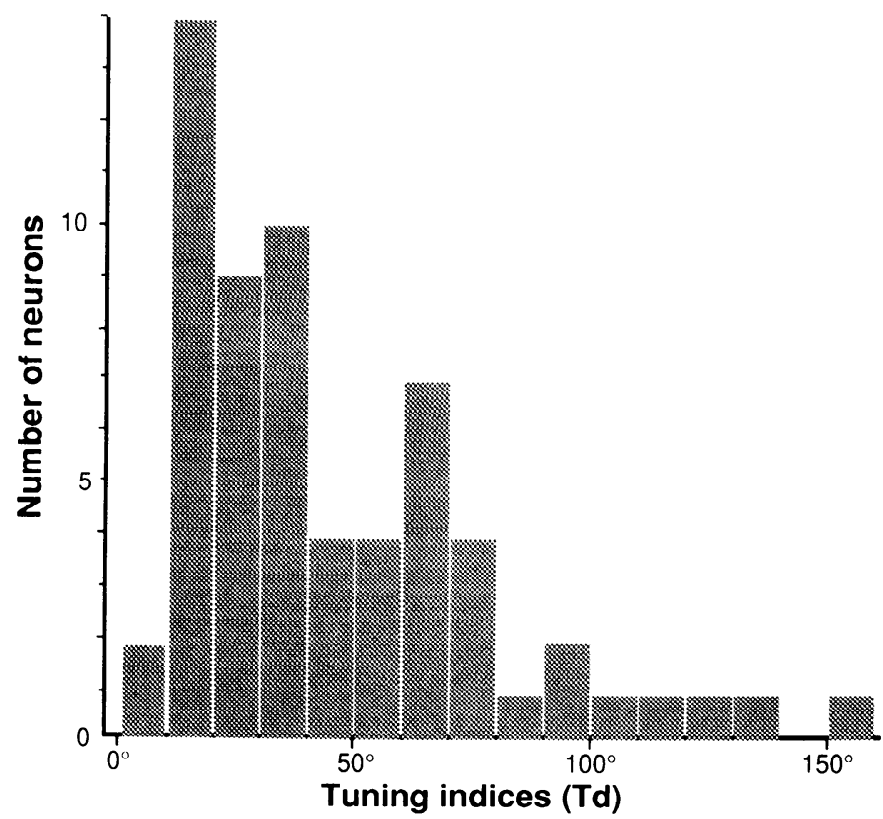

FIG. 7. Distribution of tuning indexes $\left(T_{d}\right)$ of cue-period activity in the oculomotor delayed-response task for 62 principal sulcus neurons. The mean $\pm \mathrm{SD}$ are $46 \pm 33^{\circ}$, and the median $T_{d}$ is $37^{\circ}$. tested were exactly the same as those of visual cues in the ODR task.

The VP task was applied only to neurons that had visual responses in the ODR task. No external signal was given to the monkeys when the task was changed; however, VP and ODR trials were not interspersed, but, rather, were always run in blocks of 40 or more trials.

\section{Recording and analysis of single neuron activity}

Single neuron activity was recorded with Parylene-coated tungsten microelectrodes (2-5 $\mathrm{M} \Omega$ at $1 \mathrm{kHz}$, Micro Probe) or glass-coated elgiloy microelectrodes $(0.5-1.0 \mathrm{M} \Omega$ at $1 \mathrm{kHz})$. We monitored both the raw activity signal and the window discriminator output (model DS-1, BAK) simultaneously by an oscilloscope (5110, Tektronix) and an audio monitor. After analyzing the response properties of neurons, we sometimes microstimulated through the recording electrode to determine whether the recording site was in the frontal eye fields while the monkey maintained fixation. Details of microstimulation were described in our previous report (Funahashi et al. 1989a).

The on-line computer system, in addition to carrying out the behavioral paradigms, sampled neuronal and ocular signals and stored these data in relation to task events on magnetic media. Two types of data storage files were collected. Event buffer files contained the time of every event that the computer had access to, including the time of each discriminated action potential; the time of events such as the appearance and disappearance of visual cues; and the time, duration, direction, and amplitude of each saccade made. Individual event buffer files usually contained 50-100 trials. Analog buffer files contained multiple records (1- to 2-s epochs) of eye position and velocity signals, together with the discriminated action potentials and a code representing progress through the task paradigm.

Using the stored event buffer files, we examined rasters and histograms of neuronal activity for each cue location. For the present study, the rasters and histograms were made with different alignment points including the onset of the cue and the start of the 


\section{A ODR task}

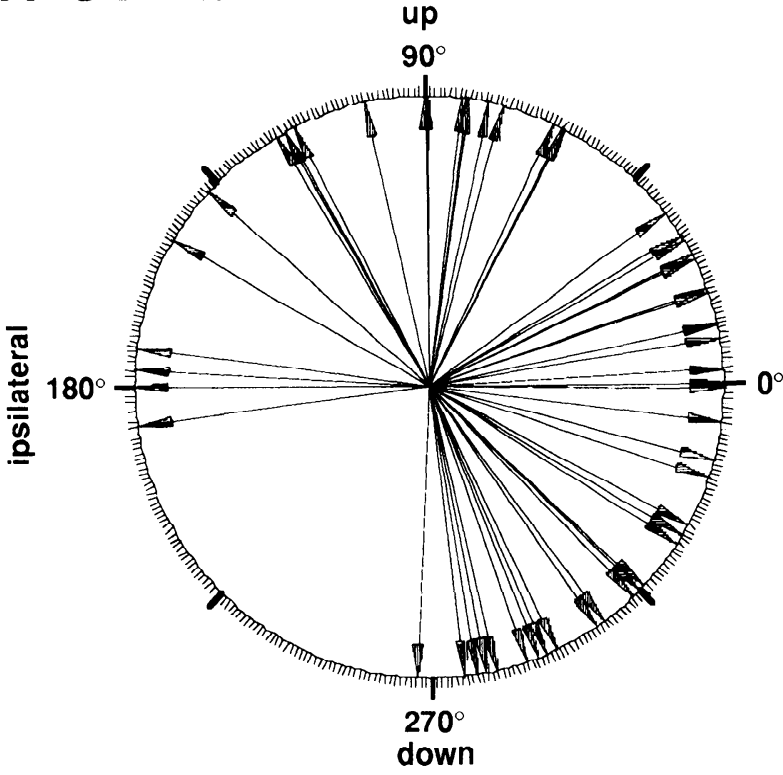

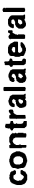

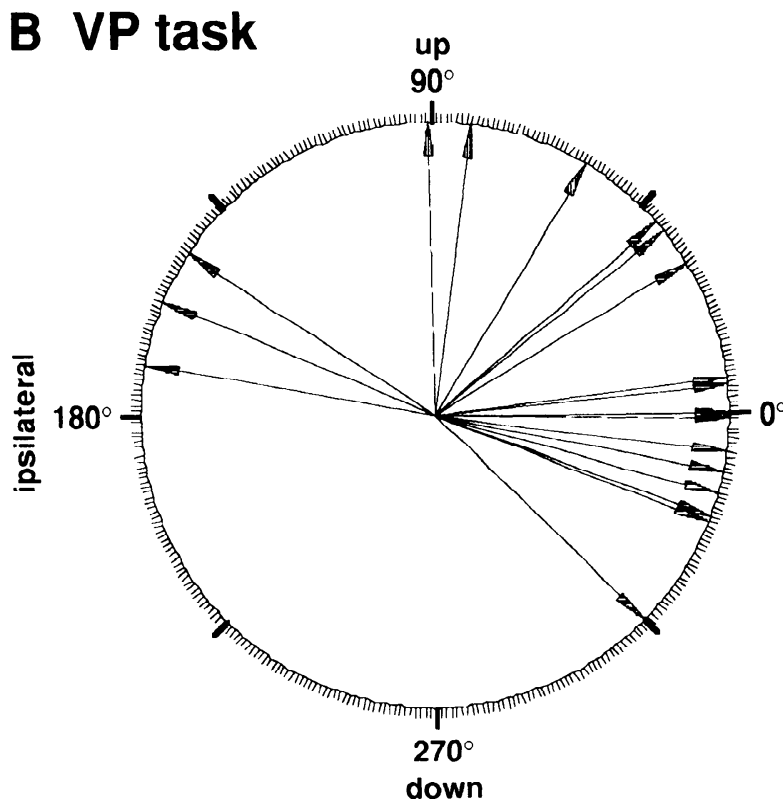

FIC. 8. A: polar plots of best directions of cue-period activity in oculomotor delayed-response (ODR) task for 62 principal sulcus neurons. $B$ : polar plots of best directions in visual probe (VP) task for 23 principal sulcus neurons. A significant contralateral bias of the best directions was observed in both tasks. Best directions were determined by parameter $(D)$ of the Gaussian function tuning curve fit. Best directions of right-hemisphere neurons were transformed into mirror-image directions in the right visual hemifield, as if all neurons were recorded from the left hemisphere.

delay period. We also made cumulative histograms to determine visual response latency at each cue location.

To analyze the directional selectivity of cue-period activity quantitatively, tuning curves of neurons that showed directional activity under the eight-cue condition were made by their best fit to the Gaussian function

$$
f(d)=B+R * \exp \left(-0.5\left\{\left[(d-D) / T_{d}\right]^{2}\right\}\right)
$$

where $f(d)$ is discharge rate as a function of cue direction $(d)$. The constants can be roughly interpreted as follows: $B$ is the spontane-

\section{A C group}

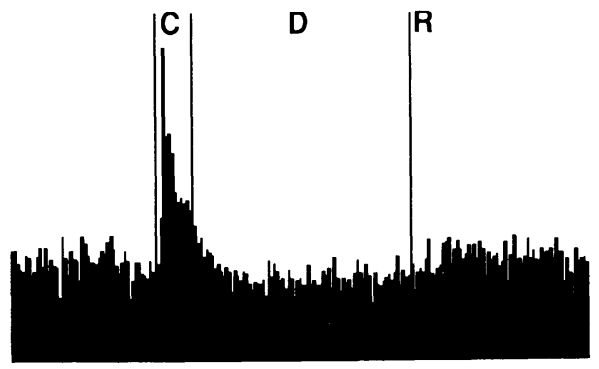

$B C+D$ group

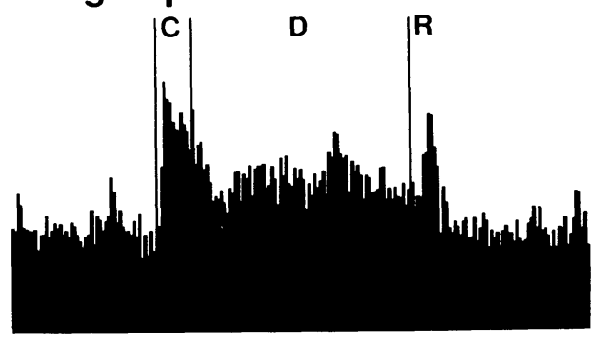

C D group

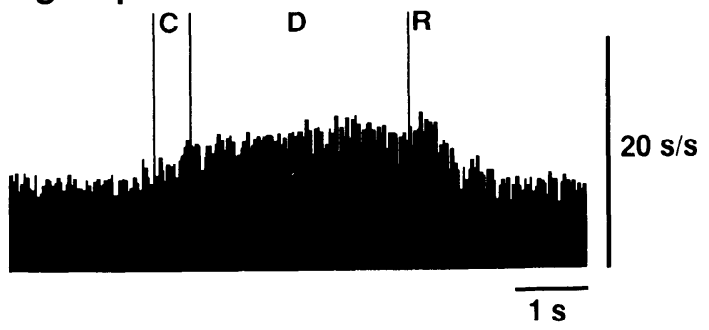

FIG. 9. Composite histograms summing over a large number of principal sulcus (PS) neurons having particular patterns of activity during oculomotor delayed-response (ODR) task. Only trials for a neuron's preferred cue direction yielding the largest response included in the composite histograms, with each neuron contributing between 7 and 12 trials. The delay duration was always $3 \mathrm{~s}$. C, D, and R indicate the cue, delay, and response periods, respectively. Histogram binwidth is $40 \mathrm{~ms}$. $A$ : composite histogram combining the activity of 27 PS neurons (216 trials) that responded during cue period but not during delay period on ODR task. $B$ : composite histogram combining activity of 33 PS neurons (272 trials) that had both cue-period and delay-period activities on ODR task. C: composite histogram combining activity of 78 PS neurons (650 trials) that had delay-period activity but no cue-period activity on ODR task.

Composite histograms contain response period activity, especially the $C+D$ group, because the classifications used to construct them ignore whether or not neurons had response period activity associated with best directions of cue and delay-period activity.

ous or background discharge rate for the neuron, $D$ is the direction yielding their best cue-period activity, $T_{d}$ is an index of tuning with respect to direction, and $R$ is the response rate at the best cue direction. This Gaussian curve method was previously used for response fields of frontal eye field neurons by Bruce and Goldberg (1985) and for memory fields of prefrontal neurons by Funahashi et al. (1989a). However, our implementation of the Gaussian formula differed from that of Bruce and Goldberg (1985), who simply fixed the offset, $B$, at the spontaneous rate of the frontal eye field neurons being studied. Instead, we allowed $B$ to vary in order to fit the PS neurons that were inhibited below spontaneous rate for cue directions opposite the best excitatory direction. The data to be fit were the maximum discharge rates over the $100-\mathrm{ms}$ portion of the $500-\mathrm{ms}$ cue period for each cue 


\section{A ODR task}
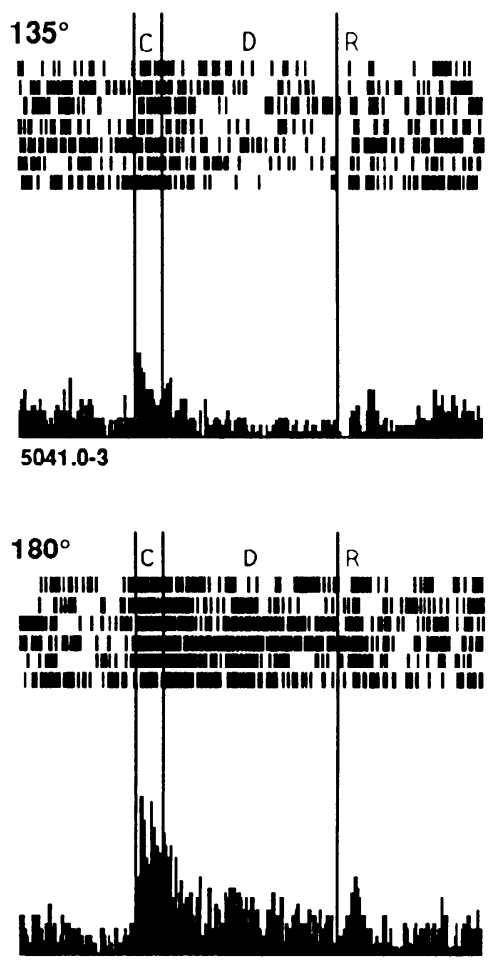

$5041.0-4$

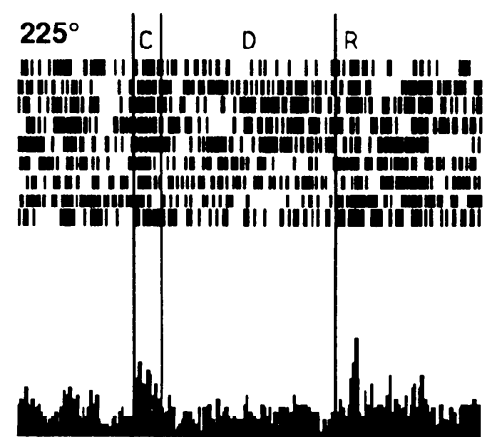

$5041.0-5$

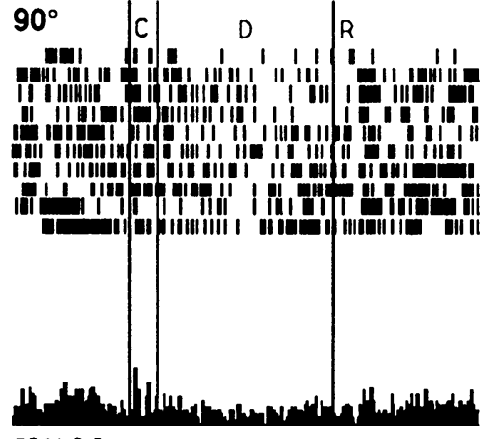

$5041.0-2$
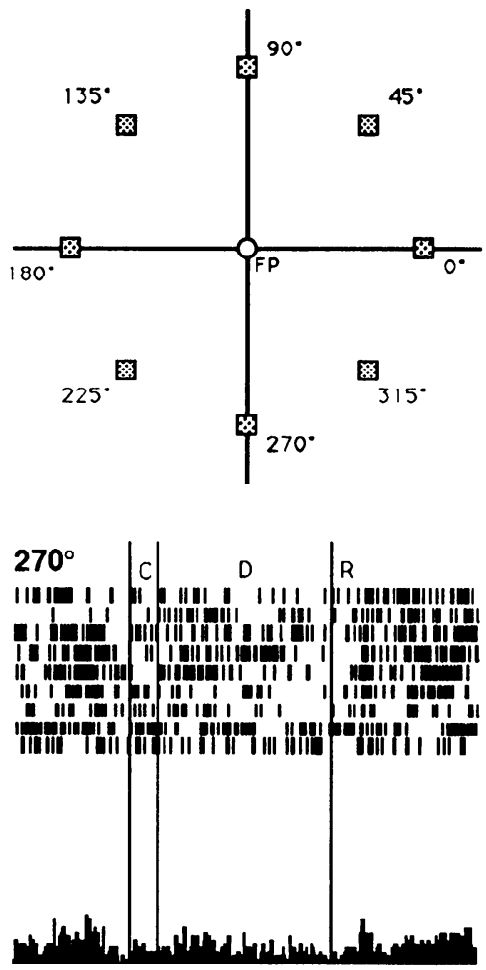

5041.0-6

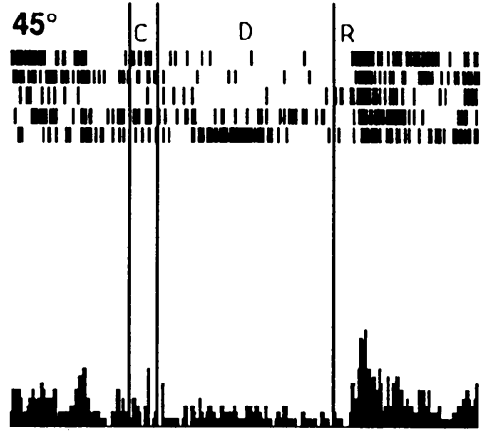

5041.0-1
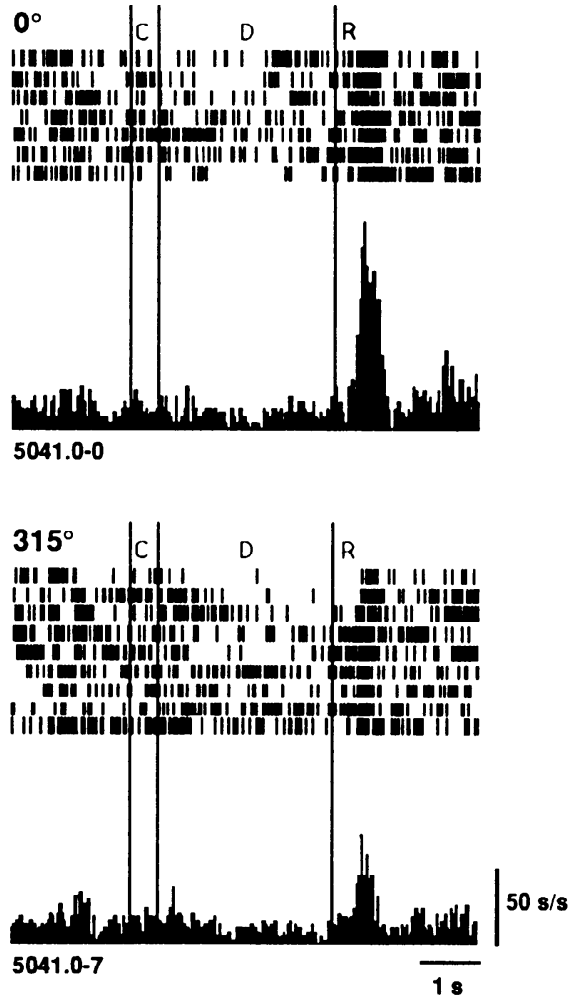

B cue period activity

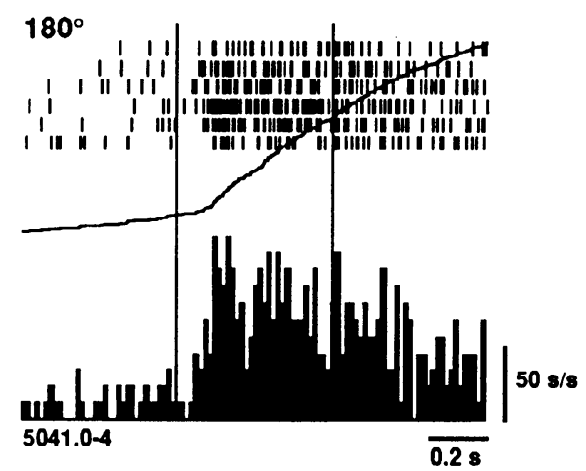

FIG. 10. Histograms demonstrating the similarity in directional preferences associated with cuc-period activity and with delay-period activity for a principal sulcus neuron (5041.0, right hemisphere) that had significant responses in both parts of the oculomotor delayed-response (ODR) task. C, D, R, cue, delay, and response periods. $A$ : both the cue- and delay-period activity were maximal after cues presented in the contralateral visual field along the horizontal meridian $\left(180^{\circ}, l e f t\right)$. At the same time, the neuron had substantial postsaccadic responses after saccades to the right (ipsilateral: 45,0 , and $315^{\circ}$ ) as well as weak presaccadic responses during saccades to the left (contralateral: 135,180 , and $225^{\circ}$ ). Visual cues were presented randomly in 1 of the 8 locations indicated in the central diagram. All cue eccentricities were $13^{\circ}$ and all delay periods were 3 s. Histogram binwidth is $40 \mathrm{~ms}$. $B$ : cue-period activity at the $180^{\circ}$ trials of same neuron $(5041.0)$ is shown with time scale expanded and a cumulative histogram in addition to rasters and conventional histogram. Visual response latency at the $180^{\circ}$ location was $80 \mathrm{~ms}$ as judged by inflection of the cumulative histogram. Histogram binwidth is $15 \mathrm{~ms}$. 


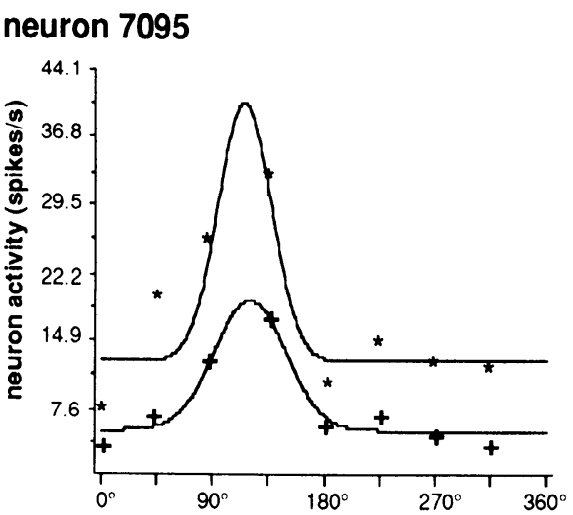

neuron 5041

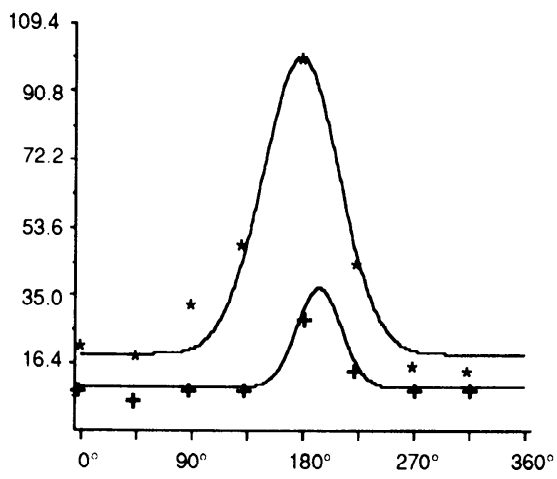

neuron 5274

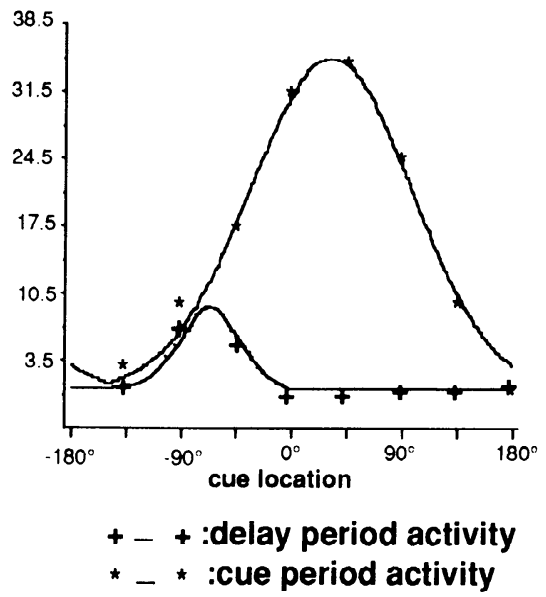

FIG. 11. Comparison of directional tuning for cue- and delay-period activities of two principal sulcus neurons having both types of response in the oculomotor delayed response task. Plots show discharge rate during the cue period and the delay period for the 8 different cue directions, with a Gaussian function fit to both sets of data. Cue-period activity for each cue dircction was measured by discharge rate during the 100 -ms period beginning at response latency of the neuron's best direction. Delay-period activity for each cue direction was the mean discharge rate during entire delay period (3 s). Neuron 7095 (left hemisphere) shows very similar tuning indexes and best directions between both activities (cue-period activity, $T_{d}=23^{\circ}$, best direction $=116^{\circ}$; delay-period activity, $T_{d}=29^{\circ}$, best direction $=121^{\circ}$ ). Neuron 5041 (right hemisphere) shows somewhat different tuning indexes and almost the same best directions between two activities (cue-period activity, $T_{d}=31^{\circ}$, best direction $=177^{\circ}$; delay-period activity, $T_{d}=18^{\circ}$, best direction $=193^{\circ}$ ). These tuning curves are based on neuronal activity shown in Fig. 10. Neuron 5274 (left hemisphere) shows fairly different best directions and tuning indexes between two activities (cue-period activity, $T_{d}=67^{\circ}$, best direction $=30^{\circ}$; delayperiod activity, $T_{d}=29^{\circ}$, best direction $=-76^{\circ}$ ). direction. The fit was implemented by iteratively searching for the $B, R, D$, and $T_{d}$ combination that minimized the least-square deviations between the data and the curve.

After 2-8 months of nearly daily recording sessions, the monkeys were sacrificed with an overdose of pentobarbital sodium and perfused with saline followed by buffered Formalin. The brains were photographed. Frozen coronal sections were taken and stained with thionin. Individual recording sites that had been marked with clectrolytic lesions $(20 \mu \mathrm{A}, 10-15 \mathrm{~s}$, tip negative for tungsten electrode and tip positive for elgiloy electrode) were identified. However, the long duration of recording and large number of electrode penetrations precluded identification of most penetrations, and their locations in the brain were estimated from their microdrive coordinates.

\section{RESULTS}

\section{General}

A total of 434 single neurons were recorded from the cortex within and surrounding the principal sulcus (PS) in 6 hemispheres of four rhesus monkeys. Among these, 261 neurons $(60 \%)$ showed excitatory or inhibitory responses in relation to one or more events of the ODR task (cue, delay, and/or response period) and were classified as task-related neurons (Fig. 2). Of 261 task-related PS neurons, 74 (28\% of task-related neurons, $17 \%$ of all PS neurons) responded to the visual cue in the cue period. The cue-period activity was defined as the response upon the presentation of the peripheral visual cues in the cue period; therefore, tonically sustained delay-period activity that often started within the cue period was excluded.

The delay-period activity of 288 among 434 PS neurons has been characterized fully in a previous report (Funahashi et al. 1989a).

\section{Characteristics of responses to visual cue stimuli}

Three types of responses to the visual cue were observed; excitatory on-responses, excitatory off-responses, and inhibitory on-responses. Most PS neurons $(n=69,93 \%)$ showed an excitatory on-response to the visual cue (Fig. $3 A)$. A few $(n=5)$ of these PS neurons had an excitatory off-response (Fig. $3 B$ ) as well. A few of the PS neurons ( $n=$ $5,7 \%$ ) showed only an inhibitory on-response (Fig. $3 C$ ).

Visual response latencies for each cue location were measured by making cumulative histograms aligned at the presentation of the cue at the locations where PS neurons had their largest response and judging the earliest point of inflection. The response latency ranged from 37 to $309 \mathrm{~ms}$ for 67 PS neurons (Fig. 4), with a mean and standard deviation $(\mathrm{SD})$ of $134 \pm 58 \mathrm{~ms}$ and a median of $116 \mathrm{~ms}$.

\section{Directional selectivity of cue-period activity}

Among 74 PS neurons with responses to visual cues, the vast majority $(n=71,96 \%)$ responded selectively or differently as a function of cue location, and, therefore, were classified as having directional cue-period activity. Only three $(4 \%)$ showed equivalent activity for all locations tested and were classified as neurons with omnidirectional cue-period activity. Figure 5 shows an example of directional cue-period activity. This PS neuron had a strong phasic response to visual cues presented in the right (con- 


\section{A Best directions (D)}

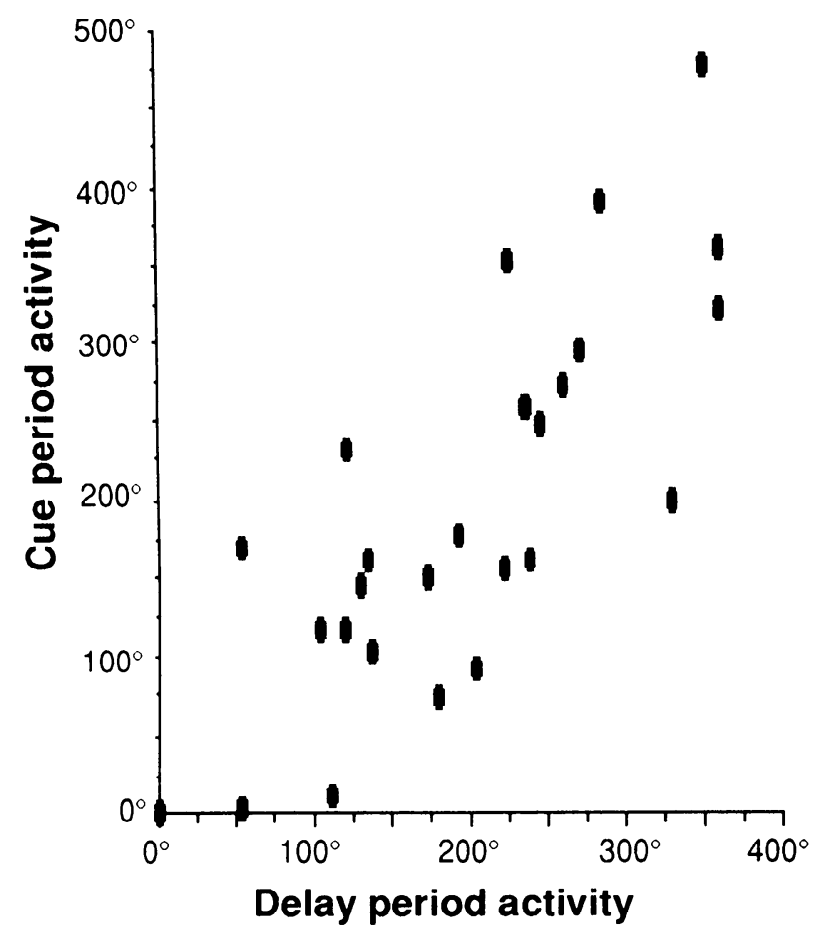

B Tuning indices (Td)

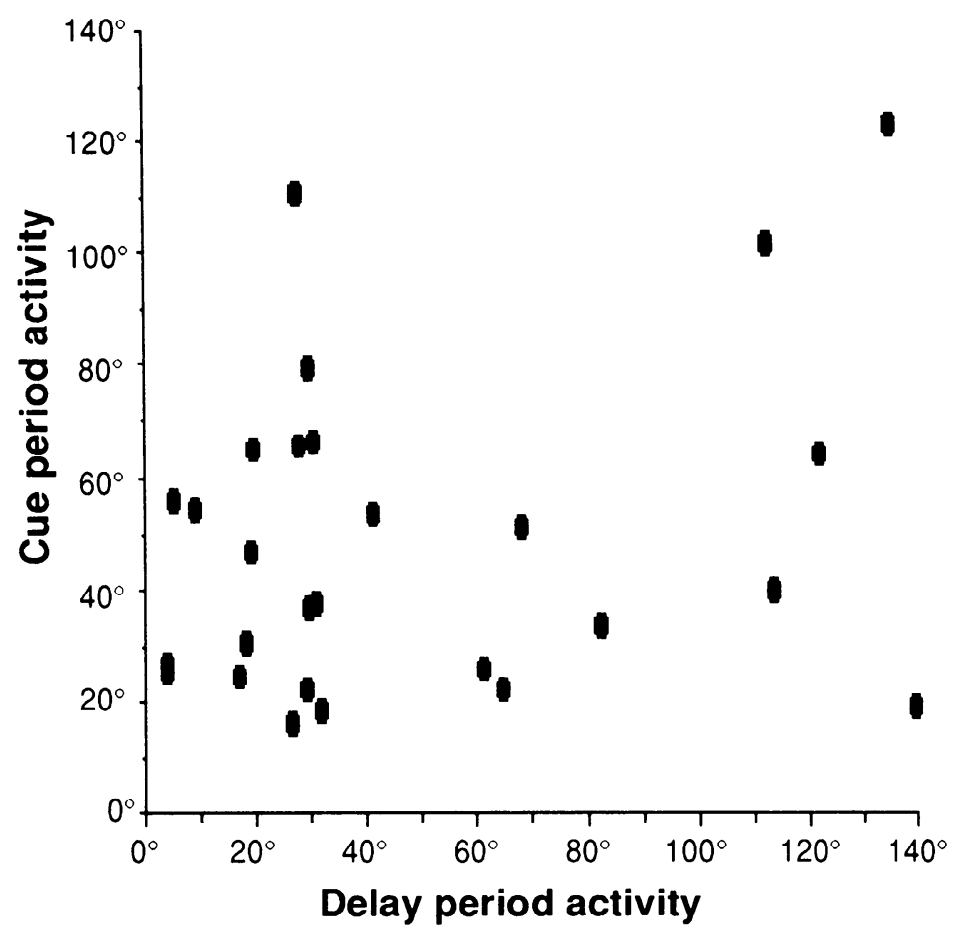

FIG. 12. Scattergrams of best directions $(D)$ and tuning indexes $\left(T_{d}\right)$ based on cue-period activity and delay-period activity in the oculomotor delayed-response (ODR) task. Both values were obtained by tuning curves for 27 PS neurons that had both activity in the ODR task and all been examined under the 8-cue condition.

tralateral) visual field, weak responses to cues in the left (ipsilateral) field, and intermediate responses to cues along the vertical meridian. The phasic response began sharply about $100 \mathrm{~ms}$ after the cue onset and ended well before the delay period commenced (Fig. 5B).

The population of directional PS neurons showed a remarkable range of tuning specificity for their visual responses in the ODR task. Some directional neurons, like the one illustrated in Fig. 5, were broadly tuned and responded to all, or nearly all, cue locations around the entire visual field, although their response magnitude varied substantially with cue directions. Other neurons responded exclusively to only one or two of the eight cue directions tested, and many neurons were intermediate in their tuning.

To quantify the directional specificity of cue-period activity, we fit cue-period activity to tuning curves defined by a Gaussian function as described in METHODS. The standard deviation parameter $\left(T_{d}\right)$ obtained from the Gaussian fit provides an index of directional specificity for each neuron. Figure 6 shows three PS neurons with different tuning functions; narrowly tuned (neuron 5277, $T_{d}=16^{\circ}$ ), moderately tuned (neuron $7074, T_{d}=37^{\circ}$ ), and broadly tuned activity (neuron 5268, $T_{d}=90^{\circ}$ ). Figure 7 shows the distribution of $T_{d}$ values for all 62 directional PS neurons that were fit; the median $T_{d}$ was $37^{\circ}$, and the mean $T_{d}$ was $47 \pm 33^{\circ}(\mathrm{SD}) . T_{d}$ values as low as $4^{\circ}$ and as high as $159^{\circ}$ were obtained. The smallest $T_{d}$ values were obtained from highly specific neurons responsive to only one direction, although their exact values are estimates because the spacing of cue directions tested was always $45^{\circ}$ for these data.
These tuning curves also provided an estimate of each neuron's best response direction ( $D$ in the formula). Figure $8 A$ shows polar plots of $D$ for 62 PS neurons in the ODR task. For analytic and graphic purposes, the best directions of right-hemisphere neurons were transformed into mirror-image directions in the right visual hemifield, as if all neurons were recorded from the left hemisphere. The majority of best directions were directed toward the visual field contralateral to the recording hemisphere; among 62 PS neurons fit with tuning curves, the best directions of 44 (71\%) were in the contralateral visual field, $11(18 \%)$ had best directions in the ipsilateral field, 4 (6\%) PS neurons had their best direction for the $90^{\circ}$ direction, and $3(5 \%)$ for the $270^{\circ}$ direction. This contralateral bias of best directions was highly significant $\left(\chi^{2}=19.800, \mathrm{df}=1, P<0.001\right)$. The upper-lower bias in the ipsilateral field evident in Fig. 8 may be caused by a sampling error because we did not observe such a bias in the best directions of the delay-period activity.

\section{Relationships between delay-period and cue-period activity in the $O D R$ task}

To study the relationships between cue- and delay-period activity, we first classified all of our PS samples solely with regard to the presence or absence of cue-period activity and delay-period activity, thereby collapsing the detailed data base presented in Fig. 2 by ignoring response period activity. This classification emphasizes the fact that many PS neurons have delay-period activity in the absence of cue-period activity, and vice versa. This yields three 


\section{A ODR task}
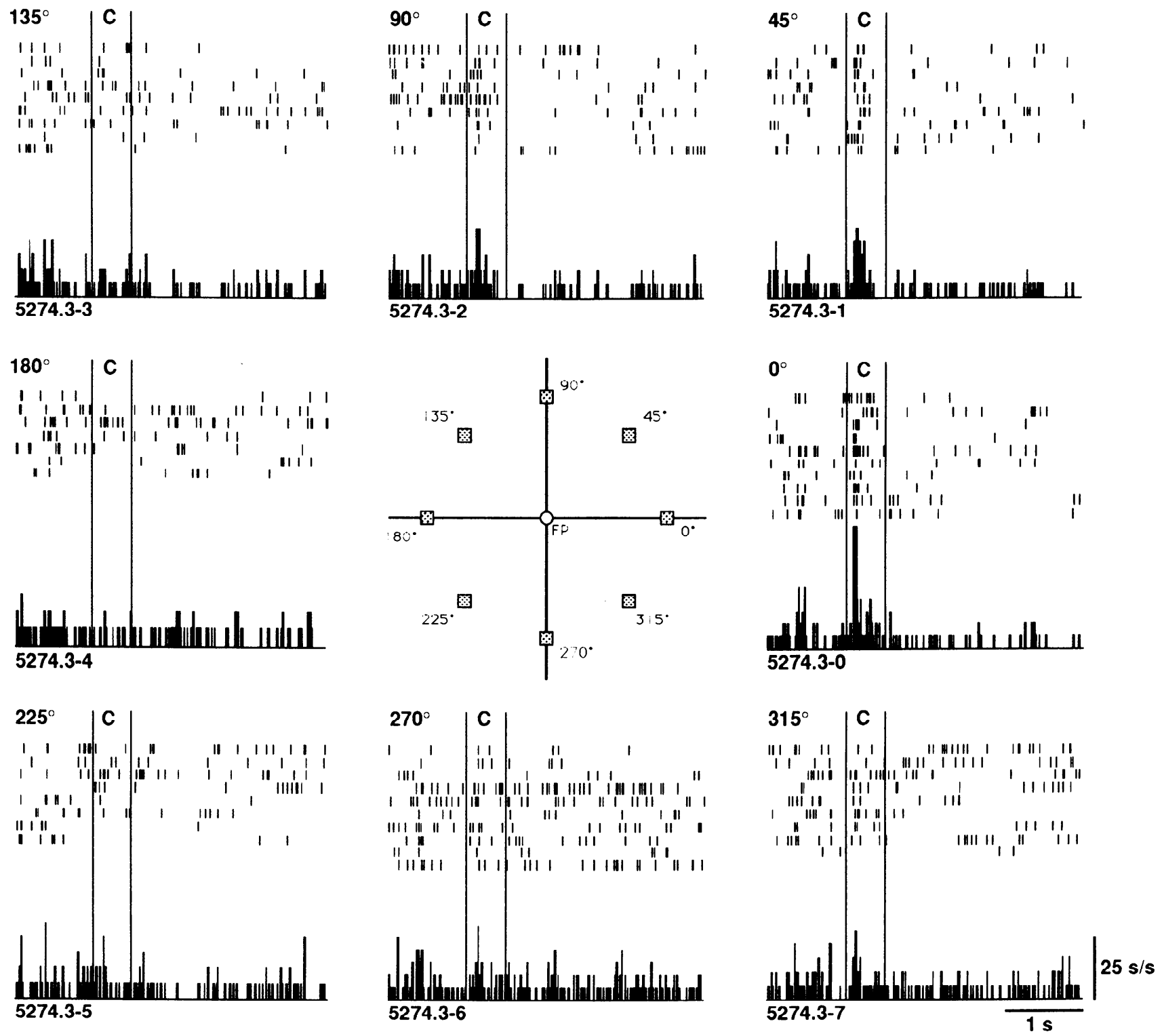

FIG. 13. A: directional cue-period activity of a principal sulcus neuron during the oculomotor delayed-response (ODR) task. This neuron (5274, left hemisphere) had phasic excitatory responses to cue stimuli presented at the 0,45 , and $90^{\circ}$ directions. Histogram binwidth is $20 \mathrm{~ms}$. $B$ : neuron's responses to same visual stimuli during visual probe (VP) task. Again, there was phasic excitatory responses to visual stimuli presented at the $0,45,90$, and $315^{\circ}$ locations. In both tasks, visual cues were randomly presented in 1 of the 8 locations indicated in the central diagram and all cue eccentricities were $13^{\circ}$. Delay period in the ODR task was $3 \mathrm{~s}$. Visual response latency at the $0^{\circ}$ location was $98 \mathrm{~ms}$ in the ODR task as well as in the VP task. Histogram binwidth is $20 \mathrm{~ms}$.

groups: a $C$ group $(n=31)$, neurons with cue-period activity in the absence of delay-period activity; a $D$ group $(n=$ 125 ), neurons with delay-period activity in the absence of cue-period activity; and a $C+D$ group $(n=43)$, neurons with cue-period activity and delay-period activity. The number of $\mathrm{D}$ neurons compared with $\mathrm{C}$ or $\mathrm{C}+\mathrm{D}$ neurons is surprisingly large.

To further explore the role of these groups and to portray their overall population activities across the PS neurons during the ODR task, we made a set of three composite histograms that added together all neuronal activities of each responsive group. These three composite histograms are shown in Fig. 9. Each neuron in groups $C, D$, and $C+D$ contributes to the appropriate composite histogram only by activity associated with the best excitatory direction for that neuron, with neurons having only inhibitory responses being excluded.

Neurons in group $C$ (Fig. 9A) show a rapid rise and an equally rapid falloff during the cue period with peak activation occurring at about $100 \mathrm{~ms}$ after cue onset. In contrast, neurons in group $D$ (Fig. $9 C$ ) show a more gradual increase, beginning after cue presentation, that reaches a plateau and then disappears abruptly in the response period. Further, although the decay time of the cue-period 


\section{B VP task}
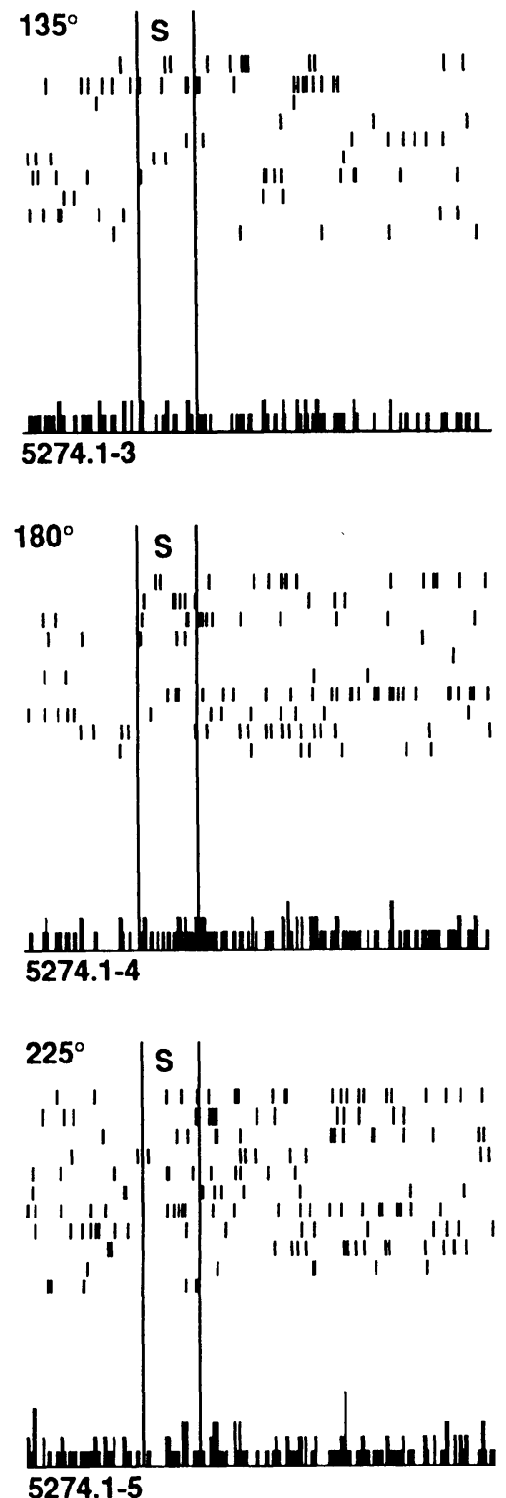
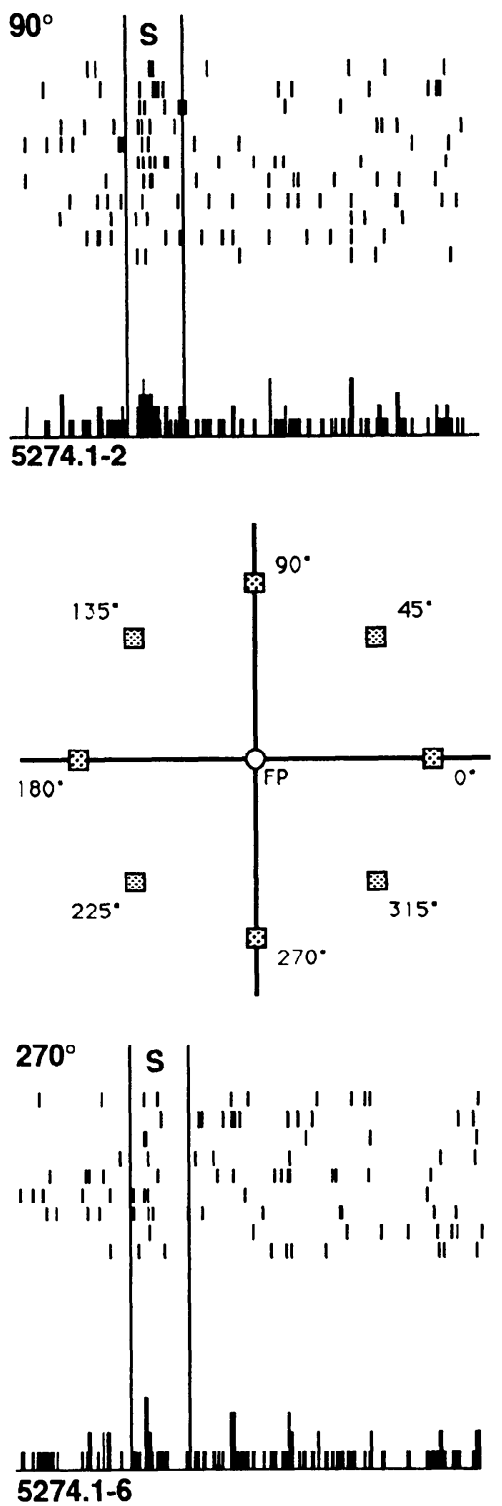
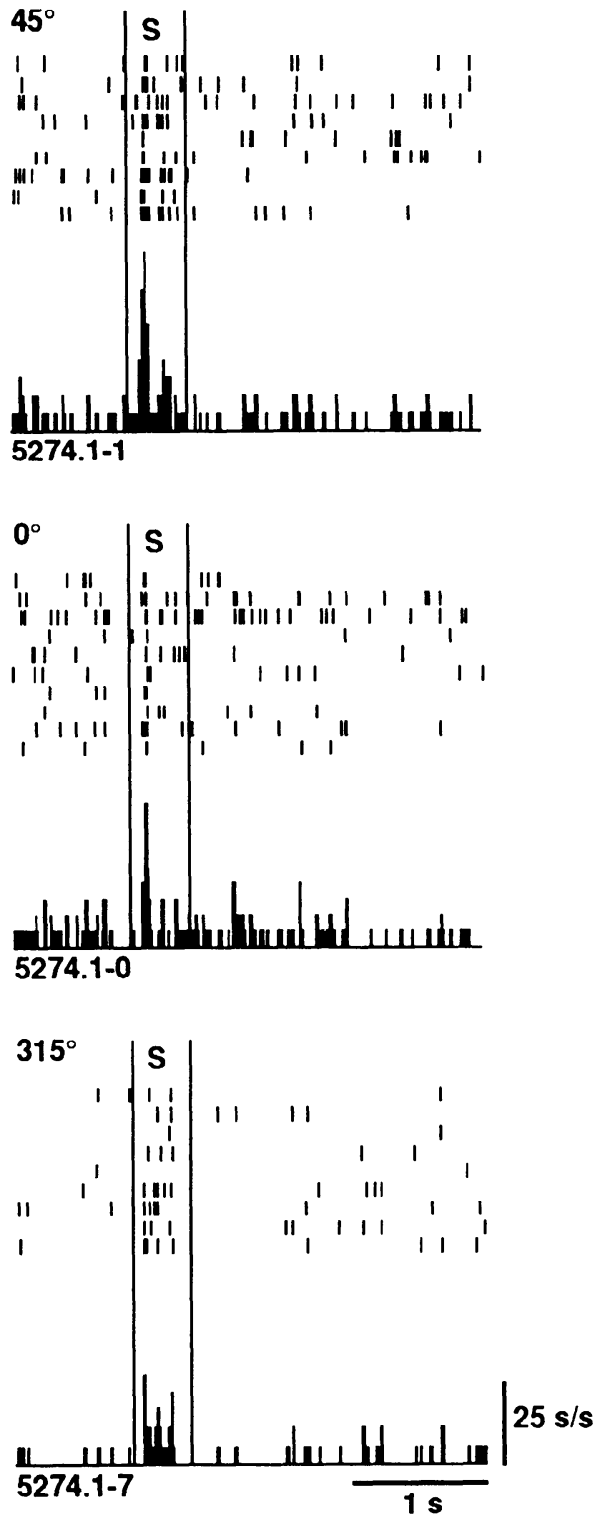

FIG. 13. (continued)

activity is a little longer, neurons in group $C+D$ (Fig. 9B) have a pattern that corresponds closely to the combined activities of group $C$ and $D$ neurons (Fig. 9, $A$ and $C$ ). In addition, the absence of any obvious cue-period activity in the composite histogram of group D neurons (Fig. 9C) or obvious delay-period activity in the composite histogram of group $C$ neurons (Fig. $9 A$ ) supports the implications that quite distinctive populations of PS neurons carry sensory and mnemonic information and also that some neurons express both.

We further evaluated the relationship of visual and mnemonic activity by comparing the response fields in the individual PS neurons that had both visual and delay period activities (the $\mathrm{C}+\mathrm{D}$ group). Figure 10 is an example of a PS ncuron that had its most pronounced cue-pcriod activation when the cue was presented at the $180^{\circ}$ location with a latency of $80 \mathrm{~ms}$; reduced but significant activation also occurred at the 135 and $225^{\circ}$ locations. This neuron also responded most actively at the $180^{\circ}$ location in the delay period. In addition to the cue- and delay-period activity, this neuron had a substantial postsaccadic activation after saccades to the right $\left(0,45\right.$, and $\left.315^{\circ}\right)$ and a weak presaccadic activation during saccades to the left $(135,180$, and $\left.225^{\circ}\right)$. Figure 11 shows the separate tuning curves fit to the cue and the delay-period activity of this (neuron 5041) as well as two other neurons (7095 and 5274). In neuron 7095 , the match between the peaks $(D)$ of the tuning curves for cue- and delay-period activity (116 and $121^{\circ}$, respectively) and the match between the tuning indexes $\left(T_{d}\right)$ for these two activities (23 and $29^{\circ}$, respectively) are very close. In contrast, for neuron 5041, although the match between the peaks $(D)$ of the tuning curves for cue- and delay-period activity is fairly close $\left(177\right.$ and $\left.193^{\circ}\right)$, a difference in tuning indexes $\left(T_{d}\right)\left(31\right.$ and $\left.18^{\circ}\right)$ was observed. Further, as is the case for neuron 5274, there can also be substantial differences in both tuning indexes and best di- 


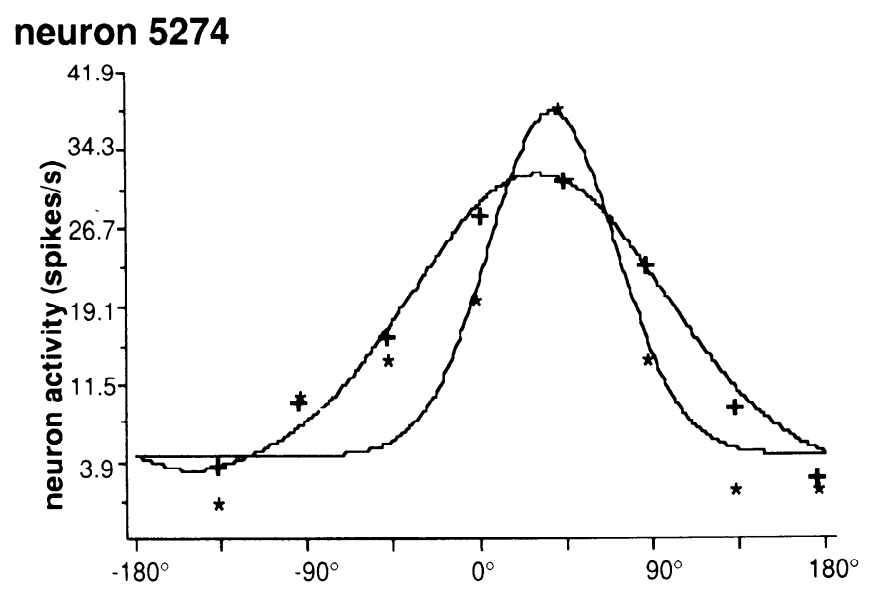

neuron 7053

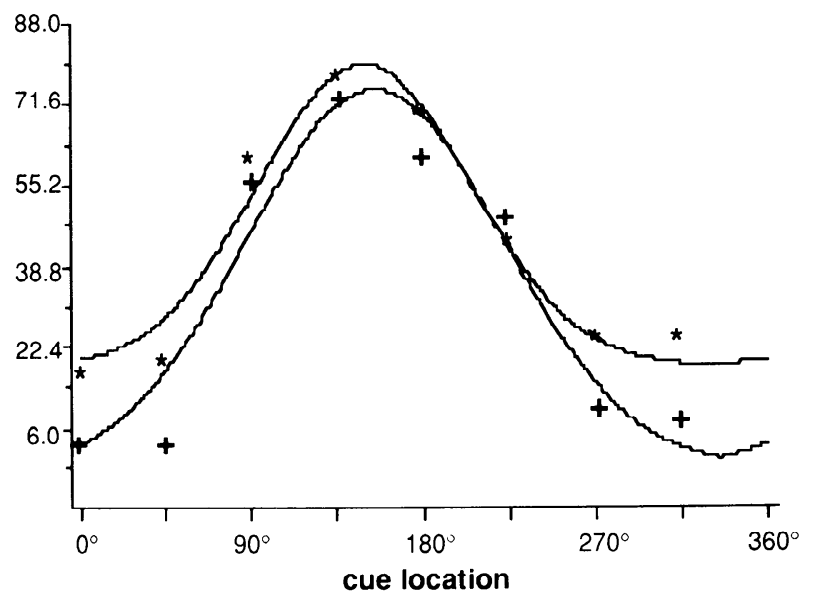

$+\ldots+$ :ODR task

* $\ldots$ : :VP task

FIG. 14. Comparison of directional tuning of cue-period activity in oculomotor delayed-response (ODR) task and of visual responses in visual probe (VP) task for two principal sulcus neurons. Plots show discharge rates for the 8 different directions, with a Gaussian function fit to both sets of data. Visual response for each cue direction was measured by discharge rate during the $100-\mathrm{ms}$ period beginning at the response latency of the neuron's best direction. Neuron 5274 (left hemisphere) has different tuning indexes but similar best directions between two tasks (ODR task, $T_{d}=66^{\circ}$, best direction $=31^{\circ}$; VP task, $T_{d}=33^{\circ}$, best direction $=38^{\circ}$ ). Neuron 7053 (right hemisphere) has very similar tuning indexes and best directions between both tasks (ODR task, $T_{d}=66^{\circ}$, best direction $=155^{\circ}$; VP task, $T_{d}=55^{\circ}$, best direction $\left.=149^{\circ}\right)$.

rections between the two types of activity (67 and $29^{\circ}$ in $T_{d}$, and 30 and $-76^{\circ}$ in $D$ ). Such tuning curve pairs were constructed for 27 PS neurons that had directional activity in both the cue and delay periods, and scattergrams (Fig. 12) and correlation coefficients of best directions and tuning indexes were used to examine the overall relationships between cue- and delay-period activity. The best directions of the cue- and delay-period activity were highly correlated $(r=0.800)$ (Fig. 12A). The tuning indexes $\left(T_{d}\right)$ for the two activities in the $\mathrm{C}+\mathrm{D}$ group were only modestly correlated $(r=0.267)$ (Fig. $12 B)$, but the mean tuning indexes $\left(T_{d}\right)$ were very close; $48 \pm 42^{\circ}$ (SD) for delay-period activity and $48 \pm 29^{\circ}$ (SD) for cue-period activity.

\section{Comparison of visual responses between the ODR task and the VP task}

To examine the extent to which the cue-period activity of a prefrontal ncuron in the ODR task simply reflects the neuron's response to visual stimuli, we compared neuronal activity in 36 PS neurons that exhibited visual responses in both the ODR and VP tasks. A typical PS neuron that had a phasic excitatory response at the 0,45 , and $90^{\circ}$ locations in both tasks is shown in Fig. 13, $A$ and $B$. The response latency was also the same in both conditions $(98 \mathrm{~ms}$ at the $0^{\circ}$ location). Thus this PS neuron's directional selectivity, response type, magnitude, and latency were virtually identical in both tasks.

Further analysis of the directional selectivity of neurons in these two tasks consisted of constructing tuning curves for 23 PS neurons that had all been examined under the eight-cue condition. The two PS neurons illustrated in Fig. 14 had very similar best directions $(D)$ and tuning indexes $\left(T_{d}\right)$ across tasks. Further, there was a strong correlation in best directions between the two tasks $(r=0.945)$ (Fig. 15A), and the polar plots of best directions also revealed a close correspondence (Fig. 8, $A$ and $B$ ). Additionally, as shown in Fig. $15 B$, there was a positive correlation $(r=0.552)$ in tuning indexes between the two tasks, and the means of the tuning indexes $\left(T_{d}\right)$ were almost identical; $45 \pm 31^{\circ}(\mathrm{SD})$ for the ODR task and $45 \pm 38^{\circ}$ (SD) for the VP task.

It is interesting that the best directions and the tuning specificity of the cue responses of PS neurons in the ODR task were more highly correlated with the best directions and tuning of their visual responses in the VP task than with those of their delay-period activity in the ODR task. This dissociation is especially impressive considering that the within-ODR correlation is derived from temporally contiguous events in the same trial, whereas the VP-visual versus ODR-cue correlation is between measures derived from two temporally separated paradigms. To further evaluate these relationships, we computed the disparity (absolute difference) among these independent measures of best direction and of directional tuning specificity for all neurons for which such comparisons could be made. The median disparity in the best direction for visual responses between the ODR and VP tasks was only $8^{\circ}(n=23)$. In contrast, the median disparity between the best direction of ODR cue-period activity and the best direction of ODR delay-period activity was $35^{\circ}(n=27)$. These two sets of disparities are significantly different (Mann-Whitney $U$ test, $U(23,27)=169, P<0.01)$. Similarly, the median disparity in directional tuning specificity was $13^{\circ}$ when tuning of visual activity were compared across the ODR and VP tasks, whereas the median disparity of tuning specificity between cue-period and delay-period activity within the ODR task was $28^{\circ}$. Again, these data significantly differ (Mann-Whitney $U$ test, $U(23,27)=297, P<0.05)$. Thus, although the directionality of the cue- and delay-period activity are much more similar than could be expected by chance, they are not as well-matched as one would predict if the delay-period activity was simply a continuation of the neuron's visual responses. 


\section{A Best directions (D)}

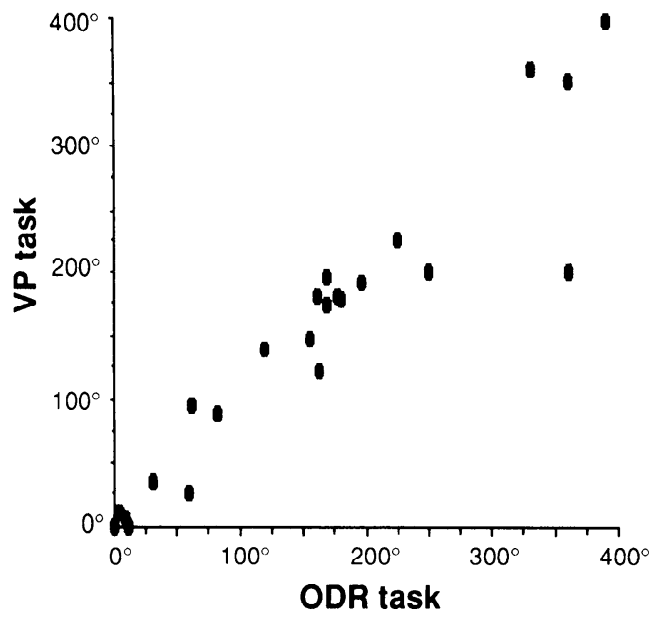

\section{Enhancement of visual response}

To examine whether an enhancement of visual responses occurs in PS neurons in the context of the ODR task relative to the VP task, we calculated an enhancement index using the following formula based on Bushnell et al. (1981)

$$
\mathrm{E}=\left(\mathrm{ODR}_{\mathrm{R}}-\mathrm{ODR}_{\mathrm{B}}\right) /\left(\mathrm{VP}_{\mathrm{R}}-\mathrm{VP}_{\mathrm{B}}\right)
$$

where $E$ is an enhancement index, $\mathrm{ODR}_{R}$ is the visual response during the cue period in the ODR task, $\mathrm{ODR}_{\mathrm{B}}$ is the baseline activity for $0.5 \mathrm{~s}$ during the fixation period of the ODR task, $\mathrm{VP}_{\mathrm{R}}$ is the visual response in the VP task, and $\mathrm{VP}_{\mathrm{B}}$ is the baseline activity for $0.5 \mathrm{~s}$ during the fixation period of the VP task. We used the peak discharge rate over $100 \mathrm{~ms}$ during the 0.5 -s cue period for $O \mathrm{OR}_{\mathrm{R}}$ and $\mathrm{VP}_{\mathrm{R}}$.

Using this formula, enhancement indexes significantly above 1.0 indicate that the behavioral manipulation augmented the sensory response. Among 34 PS neurons for which enhancement ratios were obtained, the median enhancement index was 1.12 with indexes ranging from 0.51 to 4.64 (Fig. 16). Only 7 neurons $(21 \%)$ had significant enhancement based on the cutoff value 2.0 proposed by Boch and Goldberg (1989), whereas 27 (79\%) had indexes between 0.5 and 2.0, indicating little effect of behavioral context on the visual response. Figure 16 also displays onc of the seven neurons with an enhancement index above 2.0 (neuron 5041, $\mathrm{E}=2.03$ ) and one with an index near 1.0 (neuron 5103, $\mathrm{E}=0.66$ ).

\section{Cortical distributions of neurons with cue-period activity}

Figure 17 illustrates the location of all penetrations and also that of neurons with cue-period activity in each of the five hemispheres studied. Although neurons with visual responses were distributed throughout the prefrontal cortex, more visually responsive neurons were located in the ventral and posterior paris of the prefrontal cortex. We examined cue-period activity in six hemispheres of four rhesus monkeys. However, we do not include penetration sites in the right hemisphere of monkey 2 in Fig. 17 because we could not obtain any visual response from that hemisphere. Among 305 electrode penetrations made, most were in the dorsal and ventral banks of the middle and posterior part of the PS and also in the surrounding regions of the PS. However, in two hemispheres, 18 penetrations were made in the prearcuate region bordering the anterior bank of the arcuate sulcus where the frontal eye fields are located. A total of 53 neurons were recorded from the frontal eye fields and were identified by microstimulation by the use of the same criteria $(<50-\mu \mathrm{A}$ current threshold) for evoked saccades as Bruce et al. (1985). These neurons were excluded from the data base for this report.

\section{DISCUSSION}

In the present experiment, PS neurons responded phasically to the visual stimuli in the ODR task with a median latency of $116 \mathrm{~ms}$. Virtually every PS neuron with visual responses was directionally specific, with different neurons preferring up, down, left, right, and various oblique directions and a strong overall tendency to prefer cue locations in the contralateral visual field. When neurons having cueperiod activity were tested on the VP task, they almost always responded with the same directionality, indicating that the response to the cues in the ODR task and the visual response in the VP task both reflect the same underlying visual responsivity and visual receptive field of the neuron. In addition, a comparison between the directional selectivity of cue-period activity and that of delay-period activity in the ODR task showed a strong positive correlation of the preferred directions. Such similarities between the cue- and delay-period activity suggest that the visual inputs to the prefrontal cortex play important roles in working memory processes, especially in constructing memory fields that maintain spatial information without the benefit of external cues. However, the best directions for cue- and delayperiod activity were not always identical for neurons having both activities, and, moreover, most PS neurons with delay-period activity lacked cue-period activity. Such differences indicate that the PS neuron's mnemonic activity is not derived from its sensory activity in any simple one-toone manner.

\section{Cue-period activity in the delayed-response task}

In the present experiment, the ODR task, in which the monkeys were trained to maintain fixation, and the visual 
ODR task

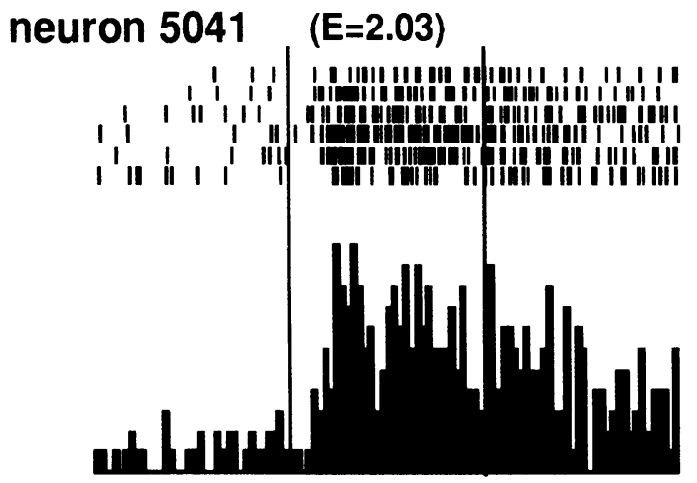

neuron $5103 \quad(E=0.66)$
VP task

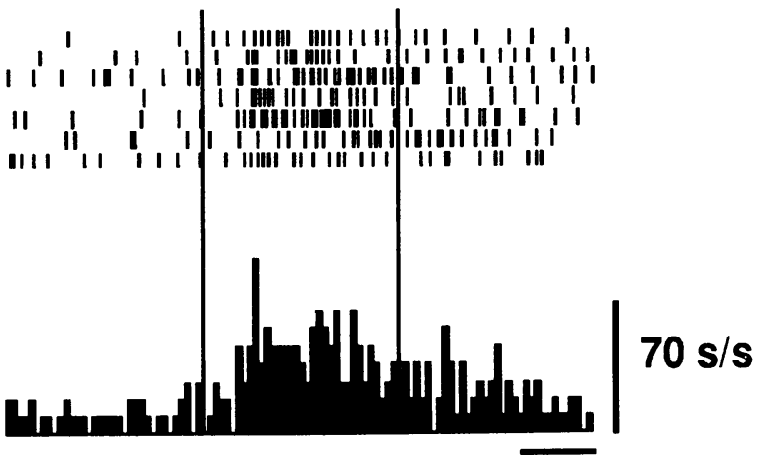

$0.2 \mathrm{~s}$

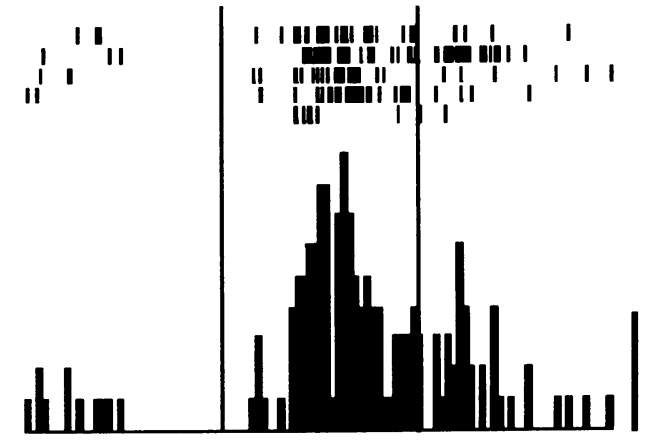

$0.2 \mathrm{~s}$

\section{Enhancement index}

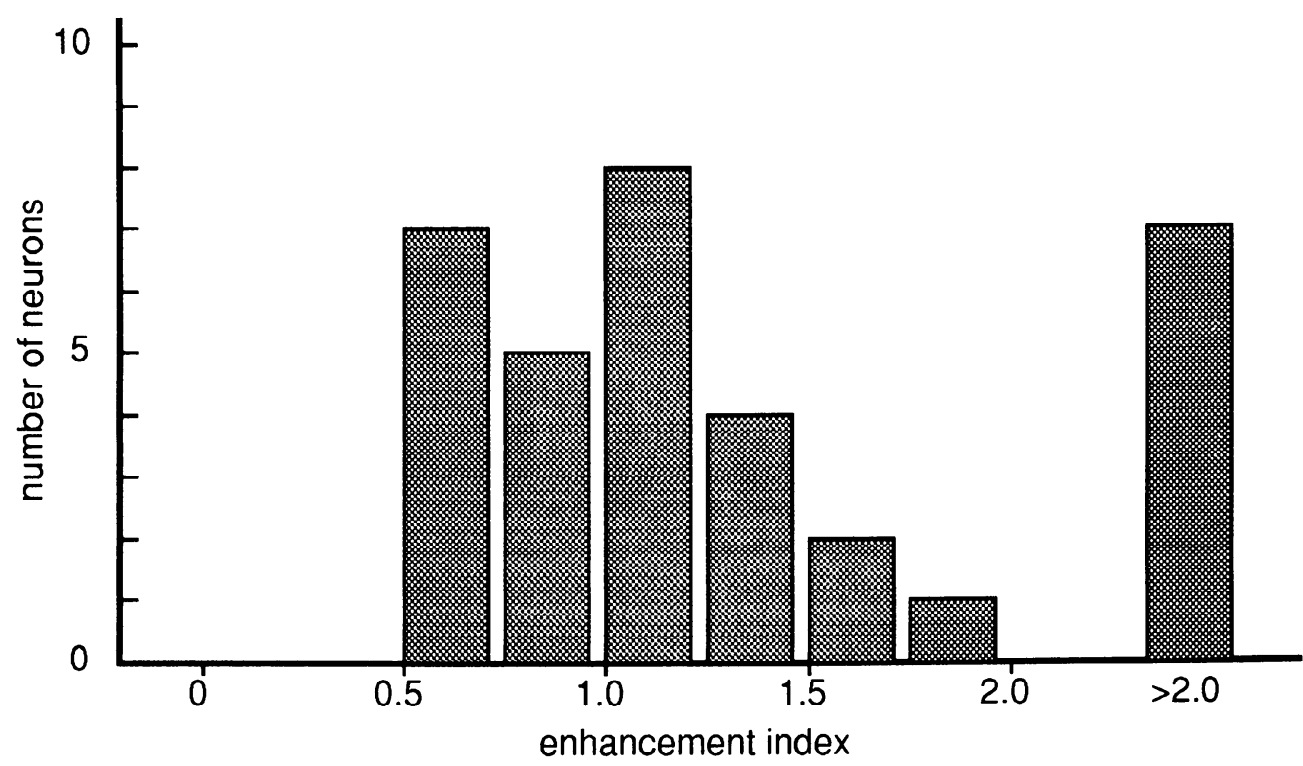

FIG. 16. Comparison of visual response in oculomotor delayed-response (ODR) task compared with that in visual probe (VP) task. Neuron 5041 is the 1 of 7 neurons with an enhancement index $>2.0(\mathrm{E}=2.03)$. Neuron 5103 is the 1 of 27 neurons with an enhancement index $<2.0(\mathrm{E}=0.66)$. Bottom bar graph shows overall distribution of enhancement indexes across the 34 PS neurons for which enhancement ratios were calculated. Mean enhancement index was $1.45 \pm 1.00$ (SD) and the median enhancement index was 1.12 . Only $21 \%(n=7)$ had significant enhancement $(E>2.0)$ with indexes ranging from 2.03 to 4.64 .

cue was randomly presented on different trials at multiple locations around the visual field, allowed us to record the monkey's direction of gaze throughout the task and, con- sequently, to know the precise retinal location of the peripheral visual cue. Using this task, we found that almost all (96\%) PS neurons with cue-period activity showed direc- 

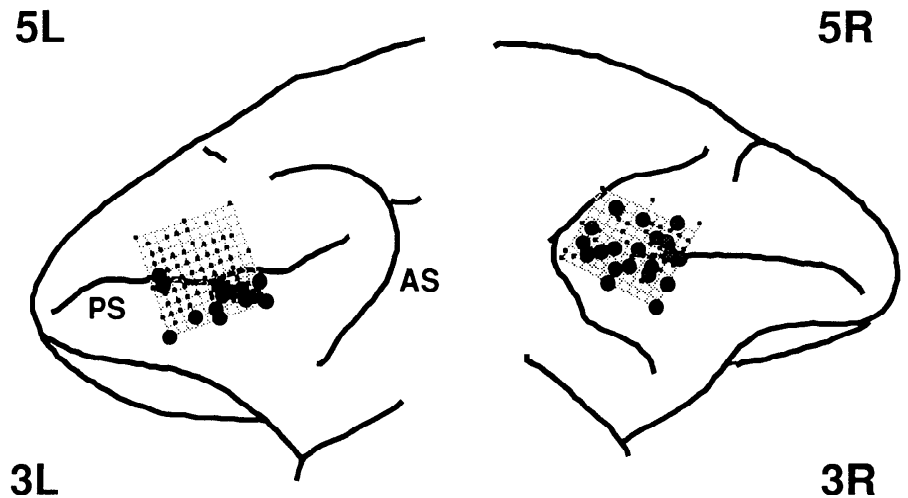

$3 L$

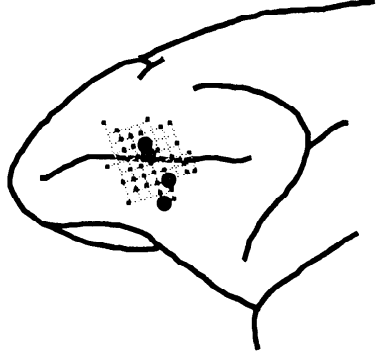

3R

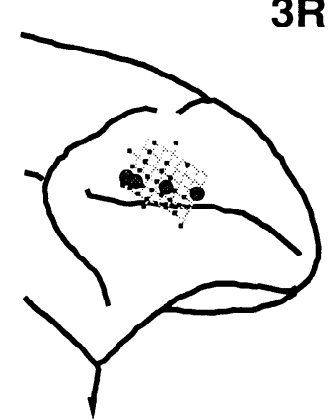

7R

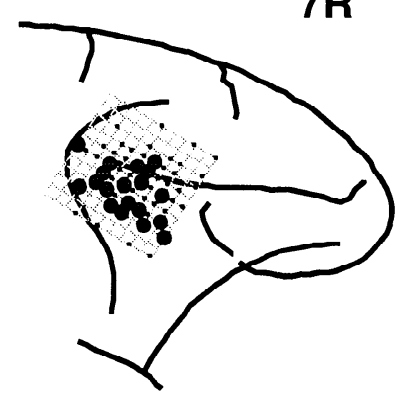

\section{Cue period activity}

FIG. 17. Tracings of dorsolateral prefrontal cortex showing location of electrode penetrations for the 3 monkeys (monkeys 3, 5, and 7) studied. Because we failed to get any visual response from the right hemisphere of monkel. 2, we exclude penetration sites of this monkey from this figure. Perspective of the tracings is approximately normal to the dorsolateral prefrontal cortical surface, roughly midway between standard lateral and dorsal views. Filled circles indicate surface locations of electrode penetrations where neurons with cue-period activity were found. PS, principal sulcus; AS, arcuate sulcus.

tional selectivity. These high percentages contrast with much lower values in previous studies. Niki (1974c), for example, reported that only $25 \%$ of prefrontal neurons with cue-period activity exhibited differential activation depending upon the left or right position of a cue in a delayed-response task. Fuster et al. (1982) reported that only $24 \%$ of all neurons with cue-period activity exhibited differential activation depending upon cue location. One explanation for a lower proportion of directional neurons in previous experiments may be the lack of an enforced fixation point in these studies, which could have allowed restricted visual receptive fields to be stimulated on both "left" and "right" trials. For example, if the monkey looked at the stimulus whenever it was presented in the cue period, a neuron with a unilateral response field bordering the fovea would have responded on every trial and, hence, been classified as nondirectional. In contrast, the ODR paradigm that we employed insured that specific retinal locations were stimulated on different trials. Even if the monkeys did not look at the stimuli in earlier studies, it is possible that broadly tuned neurons that preferred vertical or oblique stimulus locations were activated by both left and right cues and, hence, were classified as nondirectional.

Another important result concerning directional selectivity is the evidence that the neuronal response during the cue period codes not only the left-right location of visual cues but, in fact, codes arbitrary visual cue locations around the entire visual field. The same neuron was consistently activated by the same cue or set of cue locations, and different neurons coded different locations. Further, our results demonstrate that cue-period activity in the ODR task is closely related to the neuron's visual receptive field because the cue-period activity in the ODR task was similar in terms of the magnitude and tuning of response to visual responses in the VP task. Thus it appears that the directional selectivity of cue-period activity in the ODR task primarily reflects the visual receptive fields of PS neurons.

Finally, there was a strong contralateral bias in directional activity during the cue period of the ODR task, a fact not well documented or emphasized in many previous reports (Fuster et al. 1973, 1982; Niki et al. 1974c, 1976). The best direction for $71 \%$ of PS neurons with directional cueperiod activity was toward the contralateral visual field, whereas it was toward the ipsilateral field for only $18 \%$ of PS neurons. Therefore, although the prefrontal cortex receives visual information from the entire visual field, each hemisphere tends to be specialized for visual information from the visual field contralateral to it. Contralateral bias has also been noted in an oculomotor two-choice delayedresponse task by Kojima (1980) as well as by Joseph and Barone (1987). However, the failure to detect contralateral bias of cue responses during manual delayed-response tasks in many other previous studies may again reflect the lack of control of the retinal locus stimulated in delayed-response paradigms. Indeed, in conventional tests of the visual receptive field studies of PS neurons, contralateral bias is usually observed (Mikami et al. 1982a; Suzuki and Azuma 1983).

\section{Enhancement of visual responses by behavioral significance}

In the present study, only $21 \%$ of PS neurons were significantly $(E>2.0)$ enhanced by the behavioral significance of the stimulus (Fig. 16). A similar result was obtained by Mikami et al. (1982b) with the use of a visual detection task in which the monkey was required to respond to or ignore the visual stimulus depending on the instruction stimulus. In their study, only $13 \%$ of prefrontal neurons showed significant enhancement when the visual stimuli were taskrelevant. Recently, Boch and Goldberg (1989) reported that 36 and $33 \%$ of PS neurons showed significant enhancement when visual stimuli were targets of saccadic eye movements in their "synchron" and "delayed saccade" 
tasks, respectively. Furthermore, their mean enhancement indexes $(E=2.95$ in the delayed saccade task and $E=3.40$ in the synchron task) are considerably higher than ours $(E=1.45)$, especially considering that $E=1.0$ means zero enhancement. Their delayed saccade task differed from our ODR task in several important ways as follows: 1) their visual stimuli remained on during and beyond the delay interval so as to guide the delayed saccade, thus the task did not have any mnemonic requirement; 2) the "delay" between the onset of the peripheral visual stimulus and the saccade start was only 0.5 to $1.0 \mathrm{~s}$, whereas the delay in the ODR task was usually $3 \mathrm{~s}$, and sometimes $6 \mathrm{~s}$, after the offset of the cue; and 3) their peripheral target repeatedly appeared in the same location, usually in the neuron's receptive field, whereas the cue location in the ODR task was randomly varied from trial to trial. As elaborated below, the higher incidence of enhancement they found may reflect differences relative to the present study in the particular oculomotor tasks they used or in the specific region they recorded from.

The percentages of PS neurons that show enhancement in the present study are smaller than those reported for other brain structures tested under different conditions. For example, $51 \%$ of superior collicular neurons (Goldberg and Wurtz 1972), 47\% (Robinson et al. 1978) and 40\% (Bushnell et al. 1981) of posterior parietal neurons, between 40\% (Wurtz and Mohler 1976b) and 50\% (Goldberg and Bushnell 1981) of the frontal eye, fields neurons, and $42 \%$ of substantia nigra neurons (Hikosaka and Wurtz 1983) show enhancement of visual responses in conjunction with visually guided saccadic eye movements.

Several reasons may account for why PS neurons could have less enhancement than its input structures. Neurons in and around the principal sulcal have direct anatomic connections with the posterior parietal cortex (Cavada and Goldman-Rakic 1989; Goldman-Rakic and Schwartz 1982; Jacobson and Trojanowski 1977; Petrides and Pandya 1984; Schwartz and Goldman-Rakic 1984), and these pathways are a likely transcortical conduit of visual information to the prefrontal cortex. Because about one-half the visually responsive neurons in the posterior parietal cortex are not enhanced, it could be that this subset of parietal neurons project to the principal sulcal cortex, whereas the enhanced parietal neurons preferentially target the frontal eye fields. In this regard, it is of interest that the frontal eye fields and the principal sulcal area are mainly innervated by different sectors of posterior parietal cortex (Barbas 1988; Cavada and Goldman-Rakic 1989).

The smaller incidence of enhanced visual responses in our report relative to other reports could also reflect differences in the oculomotor tasks used to endow visual stimuli with behavioral significance. For example, the ODR task used in the present study separates visual responses from the execution of the saccadic eye movements by over $3 \mathrm{~s}$, whereas the visually-guided saccade task used in most previous reports elicits movements that typically begin within $200 \mathrm{~ms}$ of the visual stimulus onset. Such temporal proximity could inflate estimates in the enhancement of visual activity for neurons also having presaccadic motor activity when, in fact, their larger responses represent a summation of visual and motor activity (e.g., Hikosaka et al. 1989).
Conversely, although the ODR task requires attention to the peripheral visual cue in order to encode and remember its spatial location, it also requires suppression of the natural tendency to make a saccade immediately to the cuc. Thus, although it is plausible that visual responses of some neurons to the cues in the ODR task were enhanced in conjunction with attending to the stimulus and remembering its location, it is also functionally plausible that visual responses of other neurons were suppressed in conjunction with preventing saccades to the cues. In this regard, it is interesting that $35 \%$ of our PS neurons had enhancement indexes $<1.0$. Finally, it is also possible that the phasic activity of PS neurons to visual stimuli simply reflects the registration of sensory information, and an enhancement is not a relevant phenomenon.

\section{Construction of memory fields}

In our previous report, evidence was provided that many prefrontal neurons with delay-period activity in the ODR task had directional selectivity (Funahashi et al. 1989a). Like the results presented here, those neurons had delayperiod activity only when visual cues were presented at certain locations in the visual field, and, on the strength of this finding, we proposed that prefrontal neurons have memory fields. Analysis of the tuning of delay-period activity indicated that the majority of prefrontal neurons had their best directions toward the contralateral visual field, suggesting that memory fields were lateralized such that neurons in the right hemisphere tended to have their memory fields in the left visual field and vice versa. The finding in the present study that memory fields and the visual properties of prefrontal neurons are similar in terms of contralateral bias as well as tuning specificity suggests that a primary function of the visual input to the prefrontal cortex is in constructing the directional selectivity of the memory field. Nevertheless, it is essential to also point out that delay-period activity of PS neurons is not simply an elongation of the cue-period activity. Many PS neurons had delay-period activity in the absence of the cue-period activity. Furthermore, when neurons having both activities were analyzed, the best directions and directional specificities of their cue- and delay-period activities were not always identical (see Figs. 10-12).

The weak enhancement of the visual responses of PS neurons in the ODR task argues against a critical role of enhancement in constructing memory fields in PS neurons and, hence, in the memory process itself. This conclusion is also in accord with the fact that many neurons with memory fields do not exhibit cue-period activity, and is consistent with the vast literature that shows that lesions of the dorsolateral prefrontal cortex do not disrupt performance on a wide varicty of tasks in which sensory processing and activation are necessary (for review, see Fuster 1989; Goldman-Rakic 1987).

In fact, our results indicate that at least three groups of neurons $(C, D$, and $C+D)$ are active in the prefrontal cortex in constructing memory fields during a delayed-response task. The $\mathrm{C}$ group contains prefrontal neurons that respond phasically to the visual cues and presumably access information about the spatial location of the cue from 
other brain areas (Fig. 9A). Prefrontal neurons in the D group hold spatial information on-line as tonic delay-period activity (Fig. $9 C$ ). In the $\mathrm{C}+\mathrm{D}$ group, both kinds of activity are present, i.e., the visual response, which is phasic and decays rapidly, is followed by delay-period activity that develops gradually after the visual response has subsided (Fig. 9B). Although it is not clear how tonic mnemonic activity arises in the prefrontal cortex, one possibility is that group $C$ neurons provide the necessary spatial information to group D ncurons to initiate the mnemonic activity. At the same time, some or all neurons of the $C+D$ group might receive phasic visual inputs from the $C$ group and tonic mnemonic inputs from the $\mathrm{D}$ group, presumably by local connections within the same cortical column. Another possibility is that the $\mathrm{C}+\mathrm{D}$ group is intermediate in the construction of memory fields, i.e., the mnemonic activity of the $\mathrm{D}$ group may derive directly from the activity of the $\mathrm{C}+\mathrm{D}$ group, which, in turn, receives its input from the $\mathrm{C}$ group. Indeed, the significant positive correlation in best directions between cue- and delay-period activity in $\mathrm{C}+\mathrm{D}$ neurons is consistent with the idea that these neurons transform their sensory input into mnemonic form. At present, it is not possible to discriminate between these various alternatives, i.e., to determine whether $C, D$, and $C+D$ neurons are organized serially or in parallel. However, the close similarity of their response profiles and directional tuning indicates to us that local intercommunication between prefrontal neurons must be an important mechanism for constructing memory fields in the prefrontal cortex.

The authors thank G. Leydon for computer programs used to analyze the data quantitatively, M. Papero, S. Morgenstern, and L. Ladewig for surgical assistance and animal preparation, J. Coburn and M. Pappy for histological assistance, and $\mathbf{J}$. Musco for excellent photography. We also thank Dr. J. M. Fuster for valuable suggestions to this report.

This work was supported by National Institute of Mental Health Grants MH-38546 and MH-00298 to P. S. Goldman-Rakic, National Eye Institute Grant EY-04740 to C. J. Bruce, and by Jacob Javits Center for Excellence in Neuroscience Grant NS-22807.

Address for reprint requests: S. Funahashi, Section of Neuroanatomy, Yale University School of Medicine, 333 Cedar St., C303 SHM, New Haven, CT 06510

Received 23 June 1989; accepted in final form 6 December 1989.

\section{REFERENCES}

BARBAS, H. Anatomic organization of basoventral and mediodorsal visual recipient prefrontal regions in the rhesus monkey. J. Comp. Neurol. 276: 313-342, 1988.

Batuev, A. S., Orlov, A. A., And Pirogov, A. A. Short-term spatiotemporal memory and cortical unit reactions in the monkey. Acta Plyysiol. Acad. Sci. Ilung. 58: 207-216, 1981.

Batuev, A. S., Shaefer, V. I., AND Orlov, A. A. Comparative characteristics of unit activity in the prefrontal and parietal areas during delayed performance in monkeys. Behav. Brain Res. 16: 57-70, 1985.

BOCH, R. AND GOLDBERG, M. E. Participation of prefrontal neurons in the preparation of visually guided eyc movements in the rhesus monkey. J. Neurophysiol. 61: 1064-1084, 1989.

BRUCE, C. J. Single neuron activity in the monkey's prefrontal cortex. In: Neurobiology of Neocortex. New York: Wiley, 1988, p. 297-329.

Bruce, C. J. AND GoldberG, M. E. Primate frontal eye fields. I. Single neurons discharging before saccades. J. Neurophysiol. 53: 603-635, 1985.

Briff, C. I., Goldberg, M. E., Bushnell, M. C., and Stanton, G. B. Primate frontal eye fields. II. Physiological and anatomical correlates of electrically evoked eye movements. J. Neurophysiol. 54:714-734, 1985.
Bushnell, M. C., GoldberG, M. E., and Robinson, D. L. Behavioral enhancement of visual responses in monkey cerebral cortex. I. Modulation in posterior parietal cortex related to selective visual attention. $I$. Neurophysiol. 46: 755-772, 1981.

Cavada, C. And Goldman-Rakic, P. S. Posterior parietal cortex in rhesus monkey. II. Evidence for segregated cortico-cortical networks linking sensory and limbic areas with the frontal lobe. J. Comp. Neurol. 287: 422-445, 1989.

Funahashi, S., Bruce, C. J., And Goldman-RAkic, P. S. Mnemonic coding of visual space in monkey dorsolateral prefrontal cortex. $J$. Neurophysiol. 61: 331-349, 1989a.

Funahashi, S., Bruce, C. J., and Goldman-Rakic, P. S. Visual and mnemonic coding in the primate prefrontal cortex during oculomotor delayed-response performance. Soc. Neurosci. Abstr. 15: 786, $1989 \mathrm{~b}$.

FUSTER, J. M. Unit activity in prefrontal cortex during delayed-response performance: neuronal correlates of transient memory. J. Neurophysiol. 36: $61-78,1973$.

Fusite, J. M. The Prefrontal Cortex. New York: Raven, 1989.

Fuster, J. M., BAuER, R. H., AND JER VEY, J. P. Cellular discharge in the dorsolateral prefrontal cortex of the monkey in cognitive tasks. Exp. Neurol. 77: 679-694, 1982.

GoldberG, M. E. AND Bushnell, M. C. Behavioral enhancement of visual responses in monkey cerebral cortex. II. Modulation in frontal eye fields specifically related to saccades. $J$. Neurophysiol. 46: 773-787, 1981.

GOLDBERG, M. E. AND WURTZ, R. H. Activity of superior colliculus in behaving monkey. II. Effect of attention on neuronal responses. $J$. Neurophysiol. 35: 560-574, 1972.

GOLDMAN-RAKIC, P. S. Circuitry of primate prefrontal cortex and regulation of behavior by representational memory. In: Handbook of Physiology: The Nervous System. Bethesda, MD: Am. Physiol. Soc., 1987, vol. V, p. 373-417.

GOLDMAN-RAKIC, P. S. AND SCHWARTZ, M. L. Interdigitation of contralateral and ipsilateral columnar projections to frontal association cortex in primates. Science Wash. DC' 216: 755-757, 1982.

Hikosaka, O., Sakamoio, M., ani) Usui, S. Functional properties of monkey caudate neurons. II. Visual and auditory responses. J. Neurophysiol. 61: 799-813,1989.

HIKOSAKA, O. AND WURTZ, R. H. Visual and oculomotor functions of monkey substantia nigra pars reticulata. I. Relation of visual and auditory responses to saccades. $J$. Neurophysiol. 49: 1230-1253, 1983.

ITO, S. Prefrontal unit activity of macaque monkeys during auditory and visual reaction time tasks. Brain Res. 247: 39-47, 1982.

JaCOBSON, S. ANI TROJANOWSKI, J. Q. Prefrontal granular cortex of the rhesus monkey. I. Intrahemispheric cortical afferents. Brain Res. 132: 209-233, 1977.

JoSEPh, J. P. AND BARONE, P. Prefrontal unit activity during a delayed oculomotor task in the monkey. Exp. Brain Res. 67: 460-468, 1987.

KoJima, S. Prefrontal unit activity in the monkey: relation to visual stimuli and movements. Exp. Neurol. 69: 110-123, 1980

Kojima, S. And Goldman-Rakic, P. S. Delay-related activity of prefrontal neurons in rhesus monkeys performing delayed response. Brain Res. 248: 43-49, 1982.

KoJIMA, S. AND Goldman-RAKIC, P. S. Functional analysis of spatially discriminative neurons in prefrontal cortex of rhesus monkey. Brain Res. 291: 229-240, 1984

KOMATSU, H. Prefrontal unit activity during a color discrimination task with go and no-go responses in the monkey. Brain Res. 244: 269-277, 1982.

KuBOTA, K., IWAMOTO, I., AND SUZUKI, H. Visuokinetic activities of primate prefrontal neurons during delayed-response performance. $J$. Neurophysiol. 37: 1197-1212, 1974.

Kubota, K. And Niki, H. Prefrontal cortical unit activity and delayed alternation performance in monkeys. $J$. Neurophysiol. 34: 337-347, 1971.

Kubota, K., Tonolke, M., AND Mikami, A. Neuronal activity in the monkey dorsolateral prefrontal cortex during a discrimination task with delay. Brain Res. 183: 29-42, 1980.

Mikami, A., ITO, S., AND KubOTA, K. Visual response properties of dorsolateral prefrontal neurons during visual fixation task. J. Neurophysiol. 47: 593-605, 1982a.

MikAMI, A., ITO, S., AND KuBOTA, K. Modifications of neuron activities of the dorsolateral prefrontal cortex during extrafoveal attention. Behav. Brain Res. 5: 219-223, 1982 b. 
NIKI, H. Prefrontal unit activity during delayed alternations in the monkey. I. Relation to direction of response. Brain Res. 68: 185-196, 1974a.

NIKI, H. Prefrontal unit activity during delayed alternations in the monkey. II. Relation to absolute versus relative direction of response. Brain Res. 68: 197-204, 1974b.

NIKI, H. Differential activity of prefrontal units during right and left delayed response trials. Brain Res. 70: 346-349, 1974c.

Niki, H. AND Watanabe, M. Prefrontal unit activity and delayed response: relation to cue location versus direction of response. Brain Res. 105: 79-88, 1976.

Petrides, M. And Pandya, D. N. Projections to the frontal cortex from the posterior parietal region in the rhesus monkey. J. Comp. Neurol. 228: $105-116,1984$

Pigarev, I. N., Rizzolatti, G., and Scandolara, C. Neurons responding to visual stimuli in the frontal lobe of macaque monkeys. Neurosci. Lett. 12: 207-212, 1979.

Quintana, J., Yajeya, J., And Fuster, J. M. Prefrontal representation of stimulus attributes during delay tasks. I. Unit activity in cross-temporal integration of sensory and sensory-motor information. Brain Res. 474: 211-221, 1988.

ROBINSON, D. A. A method of measuring eye movement using a scleral search coil in a magnetic field. IEEE Trans. Biomed. Eng. 10: 137-145, 1963.

Robinson, D. L., Goldberg, M. E., and Stanton, G. B. Parietal association cortex in the primate: sensory mechanisms and behavioral modulation. J. Neurophysiol. 41: 910-932, 1978.

RoSenkilde, C. E., BAufr, R. H., AND Fister, J. M. Single cell activity in ventral prefrontal cortex of behaving monkeys. Brain Res. 209: 375-394, 1981.

SCHECHTER, P. B. AND MURPHY, E. H. Response characteristics of single cells in squirrel monkey frontal cortex. Brain Res. 96: 66-70, 1975.

SChwartz, M. L. AND Goldman-RaKic, P. S. Callosal and intrahemispheric connectivity of the prefrontal association cortex in rhesus monkey: relation between intraparietal and principal sulcal cortex. J. Comp. Neurol. 226: 403-420, 1984.

Stuss, D. T. And Bfnson, D. F. The Frontal Lobes. New York: Raven, 1986.

SUZUKI, H. AND AZUMA, M. Topographic studies on visual neurons in the dorsolateral prefrontal cortex of the monkey. Exp. Brain Res. 53: 47-58, 1983.

Watanabe, M. Prefrontal unit activity during delayed conditional discriminations in the monkey. Brain Res. 225: 51-65, 1981.

Watanabe, M. Prefrontal unit activity during delayed conditional go/no-go discrimination in the monkey. I. Relation to the stimulus. Brain Res. 382: 1-14, 1986.

WURTZ, R. H. AND MOHLER, C. W. Organization of monkey superior colliculus: enhanced visual response of superficial layer cells. $J$. Neurophysiol. 39: 745-765. 1976a.

WURT7, R. H. AND MOHI.FR, C. W. Enhancement of visual response in monkey striatc cortex and frontal eye fields. J. Neurophysiol. 39: 766-772, $1976 \mathrm{~b}$.

Yajeya, J., Quintana, J., AND Fuster, J. M. Prefrontal representation of stimulus attributes during delay tasks. II. The role of behavioral significance. Brain Res. 474: 222-230. 1988. 